\title{
Wiener Filter Based Channel Estimation for High-Speed Communication Environments
}

\author{
Ke Zhong · Xia Lei · Shaoqian Li
}

Published online: 22 May 2012

(C) The Author(s) 2012. This article is published with open access at Springerlink.com

\begin{abstract}
Wiener filter is the best channel interpolation method in terms of minimizing mean square error at the time dimension. However, Wiener filter with low order will lose its efficiency in high-speed communication environments, while Wiener filter with high order suffers from the problem of high computational complexity. In this paper, we analyze the relationships among Wiener filter with different orders and their corresponding application scenarios. Furthermore, based on the analysis, a pilot symbol design criterion for high-speed communication environments and a simplified Wiener filter based channel estimation technique are proposed, respectively.
\end{abstract}

Keywords Channel estimation · Wiener filter · High-speed communication environments $\cdot$ Long term evolution (LTE)

\section{Introduction}

With the rapid development of mobile wireless communication technology and high-speed railway construction worldwide, the moving speed of the user transceivers can be as high as $350 \mathrm{~km} / \mathrm{h}$. In this case, to ensure effective communication of future wireless technologies such as 3GPP long term evolution (LTE) [1] accurate channel parameters are indispensible. Channel parameters are essential for coherent detection, diversity combining and decoding. Therefore, implementable channel estimation method with low computational complexity

K. Zhong $(\bowtie) \cdot$ X. Lei $\cdot$ S. Li

National Key Laboratory of Science and Technology on Communications, University of Electronic Science and Technology of China, Chengdu, China

e-mail: zhongke@uestc.edu.cn

X. Lei

e-mail: leixia@uestc.edu.cn

S. Li

e-mail: 1sq@uestc.edu.cn 
that applicable to such high-speed environments has drawn remarkable interest in recent years and has remained largely an open problem to be solved urgently.

In current researches of channel estimation, when the users move at low speeds, for example less than $120 \mathrm{~km} / \mathrm{h}$, the wireless channel changes slowly and smoothly. In this case, traditional interpolation methods such as linear interpolation can be used to achieve accurate channel estimation [2-4]. However, when the users move at high speeds, such as $350 \mathrm{~km} / \mathrm{h}$ or higher, the wireless channel changes quickly and dramatically. In this case, traditional interpolation methods mentioned above cannot provide desired channel estimation performance under such high-speed environments [2-4]. As is well known, Wiener filter [5] is the best interpolation method in terms of minimizing mean square error (MMSE) at the time dimension. However, Wiener filter with low order is not applicable to channel estimation in high-speed environments, as will be demonstrated in this paper. On the other hand, Wiener filter with high order can significantly improve the performance of channel estimation in highspeed environments, at the cost of unacceptable high computational complexity due to the complex matrix inversion and Bessel function. Usually, Wiener filter with order higher than three is difficult to realize, since its computational complexity is larger than $o\left(M^{3}\right)$, where $M$ represents the orders of Wiener filter [6]. Therefore, computational complexity restricts the application of Wiener filter in high-speed environments. In current researches [4-15], the expression for Wiener filter is derived but none of them analyze the relationships among Wiener filter with different orders and neither do they illustrate the application scenarios for Wiener filter in various mobile environments, especially in high-speed cases. Furthermore, none of them present a further detailed derivation and discuss how to simplify the expression for Wiener filter, which is critical for its implementation in high-speed environments.

In order to solve the aforementioned problems and limitations of Wiener filter, in this paper we first analyze the relationships among Wiener filter with different orders and their application scenarios. Based on the analysis, a pilot symbol design criterion applicable to high-speed environments and a simplified Wiener filter technique are proposed, respectively.

The remainder of this paper is organized as follows: Sect. 2 introduces the system model and gives a review of Wiener filter based channel estimation. Section 3 first analyzes the relationships among Wiener filter with different orders and their application scenarios. Then, a pilot symbol design criterion applicable to high-speed environments and a simplified Wiener filter technique are proposed, respectively. Section 4 gives extensive simulation results that demonstrate the effectiveness of the proposed theory, criterion and technique. We conclude the paper in Sect. 5.

\section{Problem Formulation}

Let $\hat{\mathbf{P}}(k)=\left[\hat{H}_{p_{1}}(k) \hat{H}_{p_{2}}(k) \ldots \hat{H}_{p_{M}}(k)\right]^{T}$ be a column vector that represents the estimate of channel frequency response for the $k$ th subcarrier at pilot symbols in orthogonal frequency division multiple access (OFDMA) or single-carrier frequency division multiple access (SC-FDMA) system, where $p_{i}, i=1,2 \ldots, M$ represents the position of the $i$ th pilot symbol at the time dimension, $M$ represents the pilot symbol number, $k=1,2, \ldots, N, N$ represents the symbol length and $\hat{\mathbf{P}}(k)$ can be obtained by, for example, the least squares (LS) or minimum mean square error (MMSE) [16] channel estimation method. Then the channel estimate $\hat{H}_{p}(k)$ at the data symbol position $p$ can be obtained by interpolating $\hat{\mathbf{P}}(k)$. Let $\mathbf{C}_{p}(k)$ be the interpolation vector and $\hat{H}_{p}(k)$ can be obtained as 


$$
\hat{H}_{p}(k)=\mathbf{C}_{p}(k) \hat{\mathbf{P}}(k) .
$$

The expression for the interpolation vector of Wiener filter at the time dimension is obtained by minimizing the estimation mean square error $\varepsilon_{p}=E\left\{\left|H_{p}(k)-\hat{H}_{p}(k)\right|^{2}\right\}[4]$ and is given by

$$
\hat{\mathbf{C}}_{p, \text { wiener }}(k)=E\left\{H_{p}(k) \hat{\mathbf{P}}^{H}(k)\right\} E\left\{\hat{\mathbf{P}}(k) \hat{\mathbf{P}}^{H}(k)\right\}^{-1},
$$

where $E\{\cdot\}$ denotes the expected-value operator, $[\cdot]^{-1}$ denotes the matrix inversion operator, $[\cdot]^{H}$ denotes the complex conjugate transpose operator and $[\cdot]^{T}$ denotes the transpose operator. Let $\hat{\mathbf{C}}_{p \text {, wiener }}(k)=\left[\begin{array}{ll}w_{M D, 0} & w_{M D, 1} \ldots w_{M D, M-1}\end{array}\right]$ be the result of (2), where $w_{M D, m}, m=0,1, \ldots, M-1$ represent the coefficients of Wiener filter, $M D$ represents $M$ pilot symbols are adopted to complete interpolation at the time dimension.

Since the computational complexity of matrix inversion in (2) increases rapidly with the increase of $M$ [it is greater than $o\left(M^{3}\right)$ ], Wiener filter with order higher than three is difficult to realize for its high complexity. Meanwhile, as will be shown, the computation of $\hat{\mathbf{C}}_{p \text {,wiener }}(k)$ in (2) involves calculating Bessel function, which is an infinite sum formula in its original format. Therefore, we will mainly discuss the implementable Wiener filter with order less than or equal to three. The wireless channel changes slowly and smoothly when the users move at low speeds, in which case linear interpolation method [2] or Wiener filter with two orders can be adopted to interpolate the channel. However, as we shall see shortly in the next section, when the users move at very high speeds, the performance of linear interpolation method and that of Wiener filter with two orders both drop rapidly and lose their efficiency. In this case, Wiener filter with higher orders are needed to estimate the nonlinear variation of the wireless channel, which means that more pilot symbols are required.

In this paper, channel and noise are assumed to be independent from each other. Without loss of generality we assume that noise is Gaussian distribution with zero mean and variance $\sigma^{2}$. Zadoff-Chu sequence is adopted at pilot symbol $X_{p_{i}}(k)$, so that $\left|X_{p_{i}}(k)\right|^{2}=1$.

\section{Wiener Filter Based Channel Estimation}

In this section, the relationship between linear interpolation method and Wiener filter with two orders is first analyzed mathematically. Based on the analysis the conclusion that Wiener filter with two orders is not applicable to high-speed environments can be obtained. Then, the relationship between Wiener filter with two orders and Wiener filter with three orders is also analyzed mathematically, based on which the relationships among Wiener filter with different orders can be easily obtained. According to the analysis, a pilot symbol design criterion applicable to high-speed environments is also proposed in this section. Finally, a simplified Wiener filter technique is proposed based on the signal correlation and the properties of Bessel function.

For the sake of simplicity, the LS channel estimation method is employed to obtain the channel estimate at pilot symbols in the following derivations. Notice that since there is only a coefficient difference between the LS and MMSE channel estimation method for Wiener filter [15], the proposed method in this paper is not restricted to the LS channel estimation method at pilot symbols but can be easily extended to include the MMSE channel estimation method at pilot symbols. 
3.1 The Relationship Between Wiener Filter with Two Orders and Linear Interpolation Method

Let $M=2$ in (2) and after some manipulations the coefficients of Wiener filter with two orders are given by

$$
\begin{aligned}
& w_{2 D, 0}=\frac{1}{|\mathbf{A} 1|}\left[E\left\{H_{p} H_{p_{1}}^{*}\right\}\left(1+\sigma^{2}\right)-E\left\{H_{p} H_{p_{2}}^{*}\right\} E\left\{H_{p_{2}} H_{p_{1}}^{*}\right\}\right], \\
& w_{2 D, 1}=\frac{1}{|\mathbf{A} 1|}\left[E\left\{H_{p} H_{p_{2}}^{*}\right\}\left(1+\sigma^{2}\right)-E\left\{H_{p} H_{p_{1}}^{*}\right\} E\left\{H_{p_{2}} H_{p_{1}}^{*}\right\}\right],
\end{aligned}
$$

where $|\mathbf{A} 1|$ is the corresponding determinant of Wiener filter with two orders. For the sake of notational simplicity, the subcarrier index $k$ is omitted in (3), (4) and will be omitted in the following derivations. Substituting (3) and (4) into (1), the channel estimation result of Wiener filter with two orders can be expressed as

$$
\hat{H}_{2 D}=w_{2 D, 0} \hat{H}_{p_{1}}+w_{2 D, 1} \hat{H}_{p_{2}} .
$$

The expression for linear interpolation method to estimate the channel at data symbol $p$ is given by

$$
\hat{H}_{\text {linear }}=\frac{p_{2}-p}{p_{2}-p_{1}} \hat{H}_{p_{1}}+\frac{p-p_{1}}{p_{2}-p_{1}} \hat{H}_{p_{2}} .
$$

Referring to [7], for 2-D isotropic scattering, $E\left\{H_{i} H_{j}^{*}\right\}=J_{0}\left(2 \pi(i-j) f_{D} T\right)$ in (3) and (4), which represents the correlation function at the time dimension for the $i$ th and the $j$ th symbols, $f_{D} T$ is the normalized Doppler frequency, $J_{v}(z)$ is the $v$ th order Bessel function of the first kind and is defined as

$$
J_{v}(z)=\left(\frac{z}{2}\right)^{v} \sum_{l=0}^{\infty} \frac{\left(-\frac{z^{2}}{4}\right)^{l}}{l ! \Gamma(v+l+1)} .
$$

Let $v=0$ and $z=2 \pi(i-j) f_{D} T$ in (7), then $E\left\{H_{i} H_{j}^{*}\right\}$ can be expressed as

$$
J_{0}\left(2 \pi(i-j) f_{D} T\right)=\sum_{l=0}^{\infty} \frac{\left(-\pi^{2} f_{D}^{2} T^{2}(i-j)^{2}\right)^{l}}{l ! \Gamma(l+1)} .
$$

Substituting (8) into (3) and (4), then substituting the resulting (3) and (4) into (5), the relationship between (8) and (5) can be established. To establish the relationship between $\hat{H}_{2 D}$ and $\hat{H}_{\text {linear }},(6)$ is substituted into (5), letting $\sigma^{2} \approx 0$ and finally the relationship between $\hat{H}_{2 D}$ and $\hat{H}_{\text {linear }}$ can be obtained as

$$
\hat{H}_{2 D}=\hat{H}_{\text {linear }}+\frac{\left(\pi^{2} f_{D}^{2} T^{2}\right)\left(p-p_{2}\right)\left(p-p_{1}\right)}{\left(-2+\pi^{2} f_{D}^{2} T^{2}\left(p_{1}-p_{2}\right)^{2}\right)}\left(\hat{H}_{p_{1}}+\hat{H}_{p_{2}}\right)+o\left(\left(\pi^{2} f_{D}^{2} T^{2}\right)^{2}\right) .
$$

It is observed from (9) that since $\left(\pi^{2} f_{D}^{2} T^{2}\right)$ is a small number, the terms that include $\left(\pi^{2} f_{D}^{2} T^{2}\right)$ on the right-hand side of Eq. (9) is very small compared with $\hat{H}_{\text {linear }}$. Therefore, Wiener filter with two orders is approximately equal to linear interpolation method when $\sigma^{2} \approx 0$. Then a conclusion can be made that the main difference between the two is that when $\sigma^{2} \neq 0$, Wiener filter with two orders has the effect of filtering the noise, whereas 


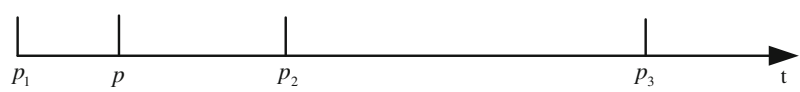

Fig. 1 The relative position of pilot and data symbols at the time dimension

linear interpolation method do not have. At the same time it is clear that in high-speed environments when the wireless channel changes dramatically and nonlinearly, Wiener filter with two orders and linear interpolation method both lose their efficiency in this situation, in which case Wiener filter with order higher than two is needed to estimate the nonlinear variation of the wireless channel.

\subsection{The Relationships Among Wiener Filter with Different Orders}

In this part, the interconversion condition between Wiener filter with two and three orders is analyzed, based on which a pilot symbol design criterion applicable to high-speed environments will be proposed.

Let $q_{i-j}=-\pi^{2} f_{D}^{2} T^{2}(i-j)^{2}$ in (8) and define a function $F\left(q_{i-j}\right)$ as

$$
F\left(q_{i-j}\right)=\sum_{l=0}^{\infty} \frac{\left(q_{i-j}\right)^{l}}{l ! \Gamma(l+1)},
$$

where $q_{p-p_{i}}=-\pi^{2} f_{D}^{2} T^{2}\left(p-p_{i}\right)^{2}$ and $q_{p_{i}-p_{j}}=-\pi^{2} f_{D}^{2} T^{2}\left(p_{i}-p_{j}\right)^{2}, i, j=1,2,3$. Then substituting (10) into (2) and letting $M=3$, after some manipulations the coefficients of Wiener filter with three orders can be obtained as

$$
\begin{aligned}
w_{3 D, 0}= & \frac{1}{|\mathbf{A} 2|}\left[F\left(q_{p-p_{1}}\right)\left(1+\sigma^{2}\right)^{2}-F\left(q_{p-p_{1}}\right) F\left(q_{p_{2}-p_{3}}\right) F\left(q_{p_{3}-p_{2}}\right)-\left(F\left(q_{p-p_{2}}\right) F\left(q_{p_{2}-p_{1}}\right)\right.\right. \\
& \left.+F\left(q_{p-p_{3}}\right) F\left(q_{p_{3}-p_{1}}\right)\right)\left(1+\sigma^{2}\right)+F\left(q_{p-p_{2}}\right) F\left(q_{p_{2}-p_{3}}\right) F\left(q_{p_{3}-p_{1}}\right) \\
& \left.+F\left(q_{p-p_{3}}\right) F\left(q_{p_{2}-p_{1}}\right) F\left(q_{p_{3}-p_{2}}\right)\right]
\end{aligned}
$$

and through similar procedure, $w_{3 D, 1}$ and $w_{3 D, 2}$ can be expressed by $F\left(q_{i-j}\right)$ as

$$
\begin{aligned}
w_{3 D, 1}= & \frac{1}{|\mathbf{A} 2|}\left[F\left(q_{p-p_{2}}\right)\left(1+\sigma^{2}\right)^{2}+F\left(q_{p-p_{1}}\right) F\left(q_{p_{3}-p_{1}}\right) F\left(q_{p_{3}-p_{2}}\right)-\left(F\left(q_{p-p_{1}}\right) F\left(q_{p_{2}-p_{1}}\right)\right.\right. \\
& \left.+F\left(q_{p-p_{3}}\right) F\left(q_{p_{3}-p_{2}}\right)\right)\left(1+\sigma^{2}\right)-F\left(q_{p-p_{2}}\right) F\left(q_{p_{3}-p_{1}}\right) F\left(q_{p_{3}-p_{1}}\right) \\
& \left.+F\left(q_{p-p_{3}}\right) F\left(q_{p_{2}-p_{1}}\right) F\left(q_{p_{3}-p_{1}}\right)\right] \\
w_{3 D, 2}= & \frac{1}{|\mathbf{A} 2|}\left[F\left(q_{p-p_{3}}\right)\left(1+\sigma^{2}\right)^{2}+F\left(q_{p-p_{1}}\right) F\left(q_{p_{2}-p_{1}}\right) F\left(q_{p_{3}-p_{2}}\right)\right. \\
& -\left(F\left(q_{p-p_{1}}\right) F\left(q_{p_{1}-p_{3}}\right)+F\left(q_{p-p_{2}}\right) F\left(q_{p_{2}-p_{3}}\right)\right)\left(1+\sigma^{2}\right) \\
& \left.+F\left(q_{p-p_{2}}\right) F\left(q_{p_{3}-p_{1}}\right) F\left(q_{p_{2}-p_{1}}\right)-F\left(q_{p-p_{3}}\right) F\left(q_{p_{2}-p_{1}}\right) F\left(q_{p_{2}-p_{1}}\right)\right]
\end{aligned}
$$

where $|\mathbf{A} 2|$ is the corresponding determinant of Wiener filter with three orders.

Figure 1 illustrates the relative position of three pilot symbols $p_{1}, p_{2}, p_{3}$ and one data symbol $p$ at the time dimension. From Fig. 1 it is observed that since $p_{3}$ is nearest to $p_{2}$, the correlation between $p_{3}$ and $p_{2}$ is critical to the performance of Wiener filter with three orders. 
From the above analysis, the relationship between Wiener filter with two and three orders can be obtained. The relationship between the determinants can be obtained as

$$
\begin{aligned}
|\mathbf{A} 2|= & |\mathbf{A} 1|\left(1+\sigma^{2}\right)+2 F\left(q_{p_{3}-p_{1}}\right) F\left(q_{p_{3}-p_{2}}\right) F\left(q_{p_{2}-p_{1}}\right)-\left(F^{2}\left(q_{p_{3}-p_{1}}\right)\right. \\
& \left.+F^{2}\left(q_{p_{3}-p_{2}}\right)\right)\left(1+\sigma^{2}\right) .
\end{aligned}
$$

The relationship between the first coefficients can be obtained as

$$
\begin{aligned}
w_{3 D, 0}= & \frac{1}{|\mathbf{A} 2|}\left[|\mathbf{A} 1| \times w_{2 D, 0}+F\left(q_{p-p_{1}}\right)\left(\sigma^{2}+\sigma^{4}\right)-\left(F\left(q_{p-p_{2}}\right) F\left(q_{p_{2}-p_{1}}\right)\right.\right. \\
& \left.+F\left(q_{p-p_{3}}\right) F\left(q_{p_{3}-p_{1}}\right)\right) \sigma^{2}-F\left(q_{p-p_{3}}\right) F\left(q_{p_{3}-p_{1}}\right)-F\left(q_{p-p_{1}}\right) F^{2}\left(q_{p_{3}-p_{2}}\right) \\
& \left.+F\left(q_{p-p_{2}}\right) F\left(q_{p_{3}-p_{2}}\right) F\left(q_{p_{3}-p_{1}}\right)+F\left(q_{p-p_{3}}\right) F\left(q_{p_{2}-p_{1}}\right) F\left(q_{p_{3}-p_{2}}\right)\right], \quad(15)
\end{aligned}
$$

and the relationship between the second coefficients can be obtained as

$$
\begin{aligned}
w_{3 D, 1}= & \frac{1}{|\mathbf{A} 2|}\left[|\mathbf{A} 1| \times w_{2 D, 1}+F\left(q_{p-p_{2}}\right)\left(\sigma^{2}+\sigma^{4}\right)-\left(F\left(q_{p-p_{1}}\right) F\left(q_{p_{2}-p_{1}}\right)\right.\right. \\
& \left.+F\left(q_{p-p_{3}}\right) F\left(q_{p_{3}-p_{2}}\right)\right) \sigma^{2}-F\left(q_{p-p_{3}}\right) F\left(q_{p_{3}-p_{2}}\right)-F\left(q_{p-p_{2}}\right) F^{2}\left(q_{p_{3}-p_{1}}\right) \\
& \left.+F\left(q_{p-p_{1}}\right) F\left(q_{p_{3}-p_{1}}\right) F\left(q_{p_{3}-p_{2}}\right)+F\left(q_{p-p_{3}}\right) F\left(q_{p_{2}-p_{1}}\right) F\left(q_{p_{3}-p_{1}}\right)\right], \quad(16)
\end{aligned}
$$

From (13)-(16) it is observed that since $\sigma^{2}$ and $\sigma^{4}$ are very small, function $F$, defined in (10), with variable $p_{3}$ in (13)-(16) will become very small and close to zero with the increase of $p_{3}$. Therefore, Wiener filter with three orders will degrade into two orders in this situation.

Now our conclusion obtained in the last paragraph will be validated through simulation. Figure 2 illustrates the relationship between $w_{3 D, i}$ and $w_{2 D, i}, i=0,1,2$ when the normalize Doppler frequency is 0.0532 (corresponding to $350 \mathrm{~km} / \mathrm{h}$ in LTE). The vertical axis represents the interpolation value and the horizontal axis is the position of $p_{3}$ at the time dimension. In the simulation we let $p_{1}=0, p_{2}=5, p=1$ in Fig. 1 and make $p_{3}$ changing from $p_{2}+1$ to 35 . From Fig. 2 it is observed that when $p_{3}$ moves at some specific point, $w_{3 D, 0}$ and $w_{2 D, 0}$, $w_{3 D, 1}$ and $w_{2 D, 1}, w_{3 D, 2}$ and 0 will converge. Also note that as $p_{3}$ increases, $w_{3 D, 0}$ and $w_{3 D, 1}$ will take small oscillation around $w_{2 D, 0}$ and $w_{2 D, 1}$, respectively, and $w_{3 D, 2}$ will oscillate around 0 . Figure 2 corroborates that under certain conditions, Wiener filter with three orders will degrade into Wiener filter with two orders. We are now in a position to investigate the factors that determine these positions of convergences.

Figure 3 illustrates the change regularity for the coefficients of Wiener filter when $p_{1}=0$, $p_{2}=5$ are fixed in Fig. 1 and moving $p$ from 1 to 4 . The solid and dotted line represent the coefficients of Wiener filter with three and two orders, respectively. From Fig. 3 a conclusion can be made that the convergences (dashed circles in Fig. 3) are not related to the relative position of $p, p_{1}$ and $p_{2}$, but are determined by the relative position of $p_{2}$ and $p_{3}$.

Then the normalized Doppler frequency is changed and continuing the simulation process. Notice that for fix wireless systems, different normalized Doppler frequencies represent different velocities. Figure 4 represents another change regularity for the coefficients of Wiener filter at 600,350 and $120 \mathrm{~km} / \mathrm{h}$ when $p_{1}=0, p_{2}=5$ and $p=1$ are fixed in Fig. 1. From Fig. 4 it is observed that not only the relative position of $p_{3}$ and $p_{2}$ affects the positions of convergences, but also the normalized Doppler frequency does. The convergences (dashed circles in Fig. 4) move to the right when velocity decreases. 


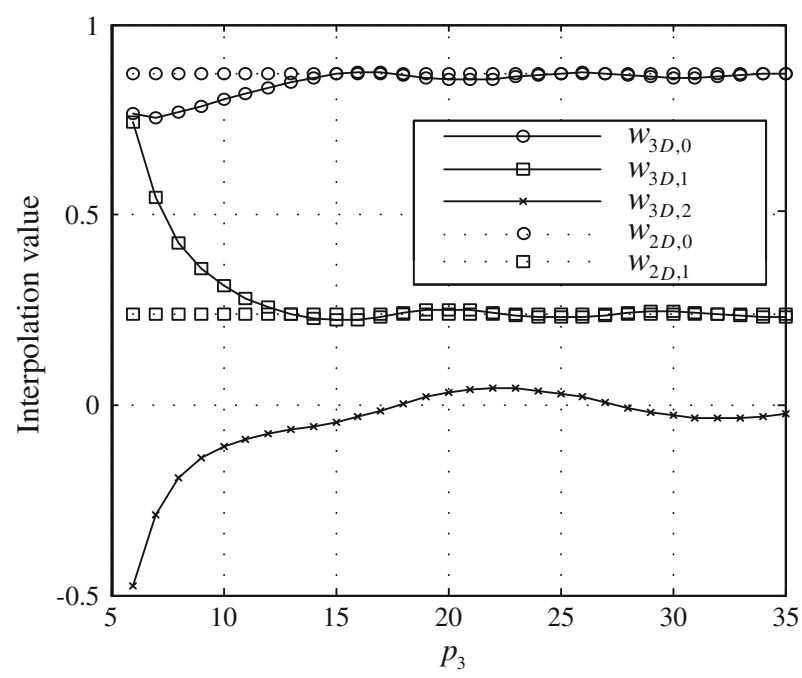

Fig. 2 The relationship between $w_{3 D, i}$ and $w_{2 D, i}, i=0,1,2$

Fig. 3 The change regularity of $w_{3 D, 0}$ and $w_{2 D, 0}$ when $p$ changes

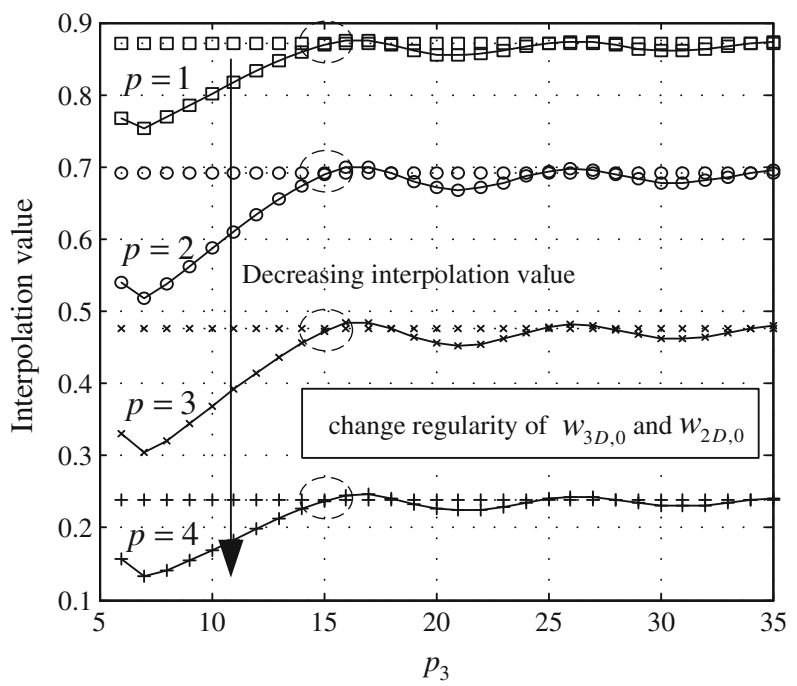

\subsection{A Proposed Pilot Symbol Design Criterion}

According to the analysis in Sect. 3.2, a final conclusion can be made that the relationship between Wiener filter with three orders and two orders are determined by the following two factors:

(1) The relative position of pilot symbols (i.e., $p_{3}$ and $p_{2}$ ).

(2) The normalize Doppler frequency $f_{D} T$.

Integrating these two factors and defining $\Delta p$ be the distance between $p_{3}$ and $p_{2}$, a conclusion can be drawn as follows: 


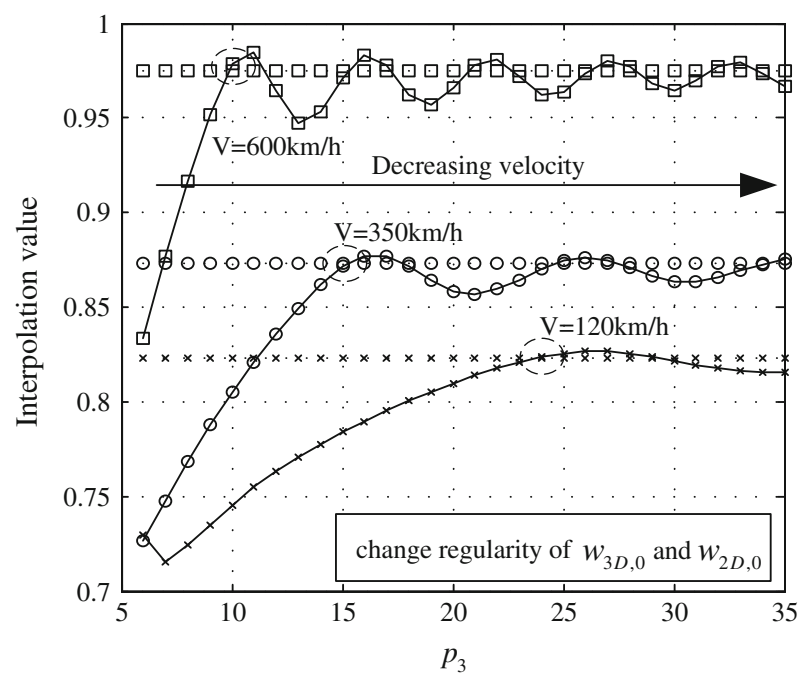

Fig. 4 The change regularity of $w_{3 D, 0}$ and $w_{2 D, 0}$ when $f_{D} T$ changes

1. If $f_{D} T \Delta p \geq 0.45$, then $w_{3 D, 0}$ will be approximately equal to $w_{2 D, 0}$. As $\Delta p$ increases, $w_{3 D, 0}$ will take small oscillation around $w_{2 D, 0}$. The reason for the oscillation is that Bessel function oscillates itself.

2. If $f_{D} T \Delta p \geq 0.35$, then $w_{3 D, 1}$ will be approximately equal to $w_{2 D, 1}$. As $\Delta p$ increases, $w_{3 D, 1}$ will take small oscillation around $w_{2 D, 1}$.

3. If $f_{D} T \Delta p \geq 0.55$, then $w_{3 D, 2}$ will be approximately equal to 0 . As $\Delta p$ increases, $w_{3 D, 2}$ will take small oscillation around 0 .

If we take the union of 1-3, the following result can be obtained that when $f_{D} T \Delta p<0.55$, the coefficients of Wiener filter with three and two orders will be not equal to each other. It can be inferred from this result that when the users move at high speeds, Wiener filter with two orders will lose its efficiency according to the analysis in Sect. 3.1. In this case, more pilot symbols need to be selected to complete Wiener filter with order higher than two in order to estimate the nonlinear variation of the wireless channel. The selection criterion for extra pilot symbols should satisfy $f_{D} T \Delta p<0.55$, otherwise there will be no performance improvement even more pilot symbols are employed, as have been proved in Sects. 3.2 and 3.3. On the other hand, the condition $f_{D} T \Delta p<0.55$ can be for sure taken as a pilot symbol design criterion that is applicable to channel estimation in high-speed environments. Apparently, it is observed that if we follow the discussion of Sects. 3.2 and 3.3, the idea of Sects. 3.2 and 3.3 can be easily and directly extended to include the analysis of the conversion condition for Wiener filter with any orders.

\subsection{A Simplified Wiener Filter Technique}

After selecting or designing extra pilot symbols according to the analysis in Sect. 3.2 and the criterion proposed in Sect. 3.3, Wiener filter with three orders is ready to be completed. However, directly using (2) when $M=3$ is still too complex for implementation in practice. In this part a simplified Wiener filter technique is proposed according to the signal correlation and the properties of Bessel function. Notice that before simplification, $F\left(q_{i-j}\right)$ in (10) is an 
Table 1 The proposed selection criterion

\begin{tabular}{ll}
\hline$q_{i-j}$ & $l$ \\
\hline$-0.2 \leq q_{i-j} \leq 0$ & $l=1$ \\
$-0.7 \leq q_{i-j}<-0.2$ & $l=2$ \\
$-1.5 \leq q_{i-j}<-0.7$ & $l=3$ \\
$-2.5 \leq q_{i-j}<-1.5$ & $l=4$ \\
$-4.2 \leq q_{i-j}<-2.5$ & $l=5$ \\
$-6 \leq q_{i-j}<-4.2$ & $l=6$ \\
$q_{i-j}<-6$ & $l \geq 7$ \\
\hline
\end{tabular}

infinite sum formula in its original format. Therefore, a simplified Wiener filter technique by selecting different $l$ in $F\left(q_{i-j}\right)$ according to different value of $q_{i-j}$ is proposed. The relationship between the proposed range of $q_{i-j}$ and $l$ in $F\left(q_{i-j}\right)$ is presented in Table 1 . The proposed selection criterion is based on the observation that $F\left(q_{i-j}\right)$ after approximation is error range of $\pm 10^{-3}$ order of magnitude compared with $F\left(q_{i-j}\right)$ in its original infinite sum formula. The detailed analysis is presented in "Appendix A".

The proposed simplified Wiener filter technique is in fact a general criterion that can be adopted to achieve reduced complexity compared with the original Wiener filter without simplification. Therefore, the approach followed to achieve simplification is detailed as follows:

1. Define $q_{i-j} \triangleq-\pi^{2} f_{D}^{2} T^{2}(i-j)^{2}$ in (8).

2. It is observed from (8) that $q_{i-j}$ determines the value of $J_{0}\left(2 \pi(i-j) f_{D} T\right)$ and thus directly affects the corresponding terms in the adopted Wiener filter, e.g., $w_{3 D, 0}, w_{3 D, 1}$ and $w_{3 D, 2}$ in (11)-(13). According to the specific problem or system we are dealing with, the value of $q_{i-j}$ can be obtained. Then, according to the proposed selection criterion in Table 1, the corresponding value for $l$ can be obtained.

3. Finally, substituting the selected value for $l$ into (8) and then substituting the resulting (8) into the adopted Wiener filter. Further the derivations and then the simplified closed-form expression for the corresponding Wiener filter can be obtained.

After approximation according to Table 1 , for a target wireless system $p_{1}, p_{2}, p_{3}$ and $p$ are fixed, the simplified $F\left(q_{i-j}\right)$ can be substituted directly into (11)-(13) and continuing the derivation to obtain the final simplified Wiener filter expression for the target wireless system. It can be observed that compared with using (2) when $M=3$ without simplification, the computational complexity can be significantly reduced according to our proposed simplified technique and moreover, it can be proved that the estimation mean square error (MSE) is also $\pm 10^{-3}$ order of magnitude compared with the original Wiener filter without approximation. The MSE derivation is detailed in "Appendix A".

\section{Simulation Results and Discussions}

In this section, extensive computer simulation results are presented to demonstrate the proposed theory, criterion and technique. LTE uplink system is chosen in our simulation [17]. Its subframe structure is given by [18] and is shown in Fig. 5. The simulation parameters are listed in Table 2. Considering that our proposed algorithms possibly require more than two pilot symbols at the time dimension, the sounding reference symbol defined in LTE uplink 
Table 2 Simulation parameters

\section{Modulation \\ Velocity}

Channel model

Transmission bandwidth

Carrier frequency

Transmit and receive antenna numbers
16QAM, 64QAM

$120 \mathrm{~km}, 350 \mathrm{~km} / \mathrm{h}$

LTE-EVA

$20 \mathrm{MHz}$

$2.3 \mathrm{GHz}$

One transmit antenna and two receive antennas

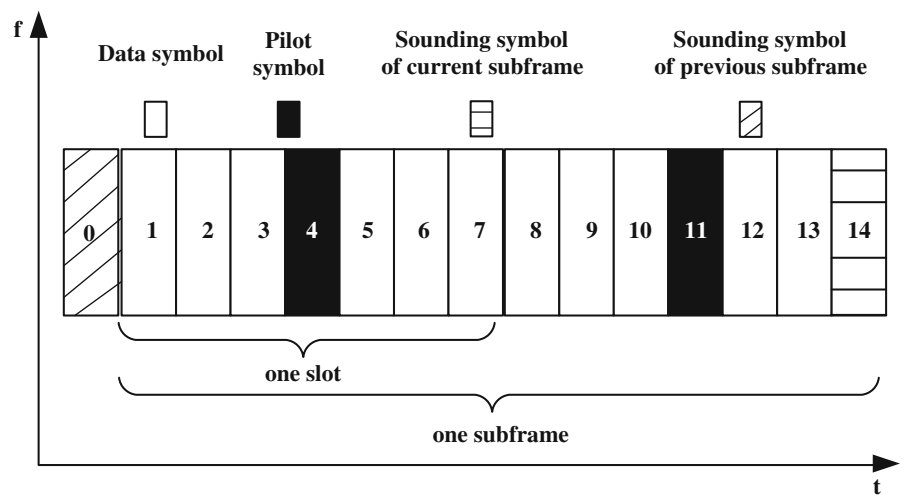

Fig. 5 The subframe structure in LTE uplink system

system is also considered according to [18]. The sounding reference symbol is used by the base station to evaluate the quality of wireless channels. It is known by the base station so that the sounding reference symbol can be regarded as extra pilot symbols to aid interpolation at the time dimension. Therefore, there are four pilot symbols in total, which consists of symbols 0, 4, 11 and 14 as shown in Fig. 5. The LS channel estimation method is adopted to obtain the channel estimate at pilot symbols in the simulation. The complete model for the simulation is shown in Fig. 6 and more specifically, for practical consideration, the coherent linear zero-forcing (ZF) equalization method is employed to mitigate the randomness of the channel.

First, the theory that established in Sect. 3.1 is validated through Fig. 7 without turbo coding. It is observed from Fig. 7 that the performance of Wiener filter with two orders is just slightly better than that of linear interpolation method at $120 \mathrm{~km} / \mathrm{h}$, for the reason that the wireless channel changes slowly and linearly in this situation. Figure 7 also shows that at $350 \mathrm{~km} / \mathrm{h}$, in which case the wireless channel changes nonlinearly and dramatically, Wiener filter with two orders is not enough to interpolate channel at the time dimension for its bit error rate (BER) is bigger than 0.1 .

Next, the pilot symbol design criterion that proposed in Sect. 3.3 is validated through Fig. 8 without turbo coding. In Fig. 8, pilot symbols 0,11 and 14 are selected to interpolate data symbols 12 and 13. Compared with only adopting pilot symbols 11 and 14 to interpolate data symbols 12 and 13, it is observed that the performance of the two schemes are almost the same because $f_{D} T \Delta p=0.58>0.55$ in this situation. However, when pilot symbols 4 , 11 and 14 are selected to interpolate data symbols 12 and 13 , in which case $f_{D} T \Delta p=0.37$, there is about $2 \mathrm{~dB}$ signal-to-noise ratio (SNR) gain compared with the case where only 


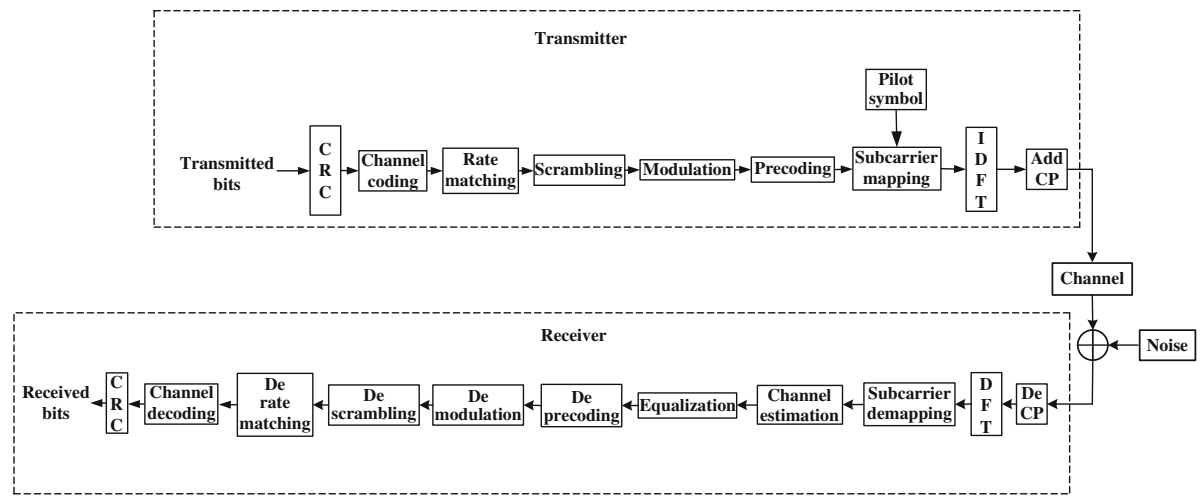

Fig. 6 The complete model for the simulation, where $C R C$ cyclic redundancy check; IDFT inverse discrete Fourier transform; $C P$ cyclic prefix; DFT discrete Fourier transform

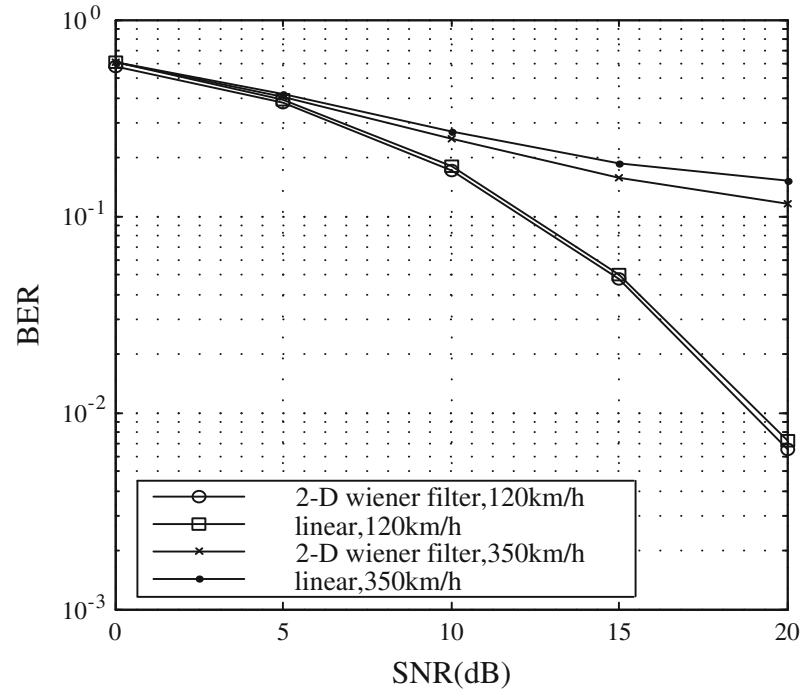

Fig. 7 Performance of BER: 64QAM, 120 and $350 \mathrm{~km} / \mathrm{h}$

pilot symbols 11 and 14 are adopted to interpolate data symbols 12 and 13. Furthermore, when pilot symbols 4,11 and 14 are selected to interpolate data symbol 8, more than $10 \mathrm{~dB}$ SNR gain can be obtained compared with the case where only pilot symbols 4 and 11 are adopted to interpolate data symbol 8 , in which case $f_{D} T \Delta p=0.16$. It is demonstrated that as long as $f_{D} T \Delta p<0.55$ is satisfied, Wiener filter with higher orders will significantly improve the accuracy of channel estimation. However, when $f_{D} T \Delta p>0.55$, Wiener filter with higher orders will have no or just trivial improvement than Wiener filter with low orders.

Finally, according to the extra pilot symbol selection criterion proposed in Sect. 3.3, data symbols 1-3, 5-7 are interpolated by pilot symbols 0,4 and 11 . Data symbols $8-10$, 12-13 are interpolated by pilot symbols 4,11 and 14 . Notice that there are 33 coefficients of Wiener filter with three orders need to be estimated in total. The details of how to imple- 


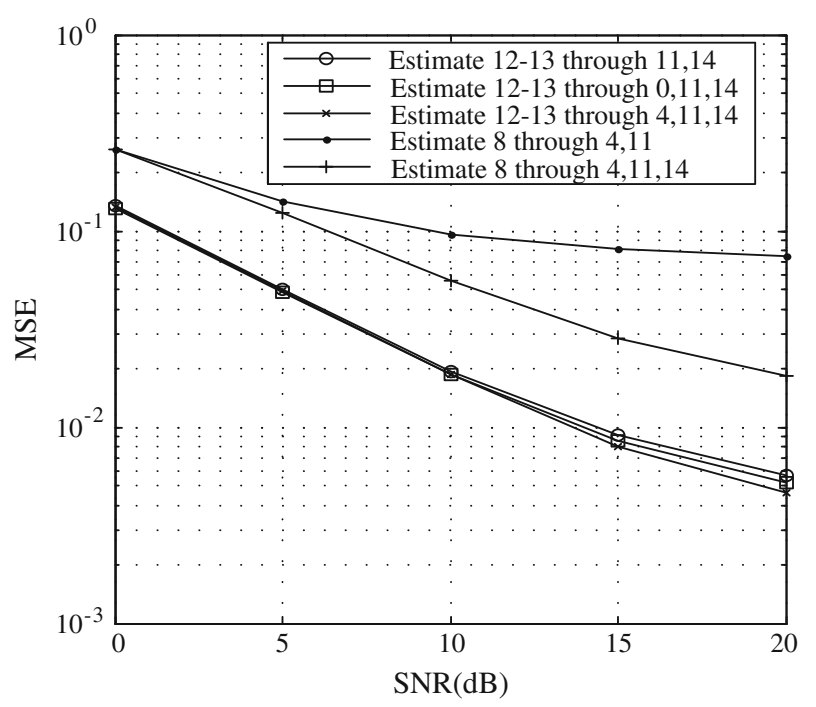

Fig. 8 Performance of MSE: 16QAM, $350 \mathrm{~km} / \mathrm{h}$

Table 3 The computational complexity comparision

\begin{tabular}{llllll}
\hline Method & \multicolumn{2}{l}{$\begin{array}{l}\text { Number of multiplication } \\
\text { per subframe }\end{array}$} & & \multicolumn{2}{l}{$\begin{array}{l}\text { Number of addition per } \\
\text { subframe }\end{array}$} \\
\cline { 2 - 3 } $\begin{array}{lllll}120 \mathrm{~km} / \mathrm{h} \\
l=2\end{array}$ & $\begin{array}{l}350 \mathrm{~km} / \mathrm{h} \\
l=6\end{array}$ & & $\begin{array}{l}120 \mathrm{~km} / \mathrm{h} \\
l=2\end{array}$ & $\begin{array}{l}350 \mathrm{~km} / \mathrm{h} \\
l=6\end{array}$ \\
\hline $\begin{array}{l}\text { The proposed method } \\
\text { The original Wiener filter with three orders }\end{array}$ & 858 & 504 & & 241 & 401 \\
\hline
\end{tabular}

ment the simplified Wiener filter technique proposed in Sect. 3.4 are shown in "Appendix B".

Comparing the simplified expression obtained in "Appendix B" with the original Wiener filter without simplification shown in (2), it is observed that our proposed simplified Wiener filter technique is much simpler than the original Wiener filter. The computational complexity comparison is shown in Table 3. Through simple computation it is obvious to conclude that when $l=6,63.64 \%$ number of multiplication per subframe and $42.41 \%$ number of addition per subframe can be saved. When $l=2,60.61 \%$ number of multiplication per subframe and $43.82 \%$ number of addition per subframe can be saved. In total, the overall computational complexity is significantly reduced through our proposed technique.

Figures 9, 10, 11, 12, 13 illustrate the performance of mean square error (MSE) and BER with regard to SNR without turbo coding. Figures 9, 10 verify that when the users move at $120 \mathrm{~km} / \mathrm{h}$, the performance of Wiener filter with three orders is only slightly better than that of Wiener filter with two orders. There is only about less than $0.5 \mathrm{~dB}$ SNR gain for the reason that the wireless channel changes slowly and linearly when the users move at low speeds. It is noted that the performance of our proposed simplified Wiener filter technique is almost the same as that of the original Wiener filter without simplification. 


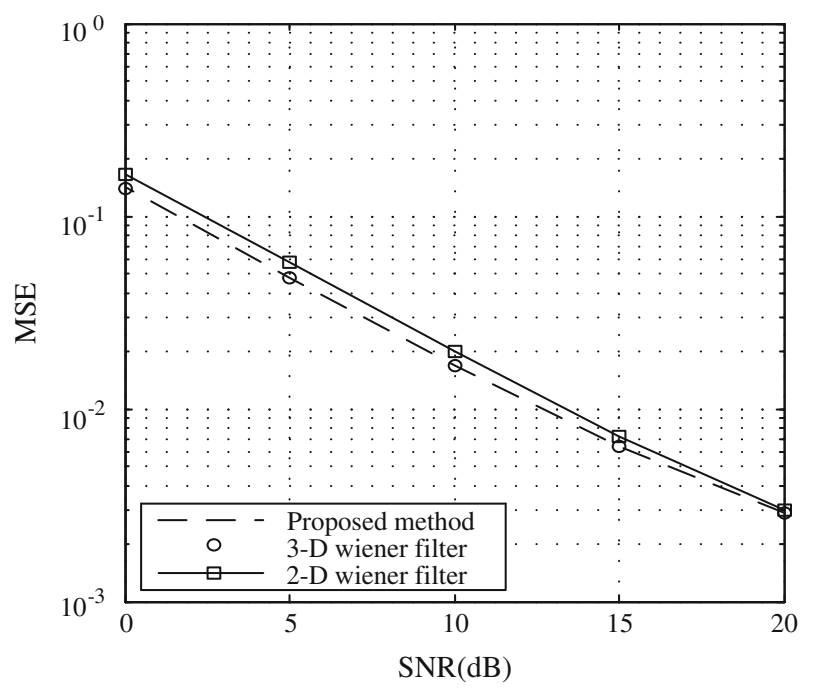

Fig. 9 Performance of MSE: 64QAM, $120 \mathrm{~km} / \mathrm{h}$

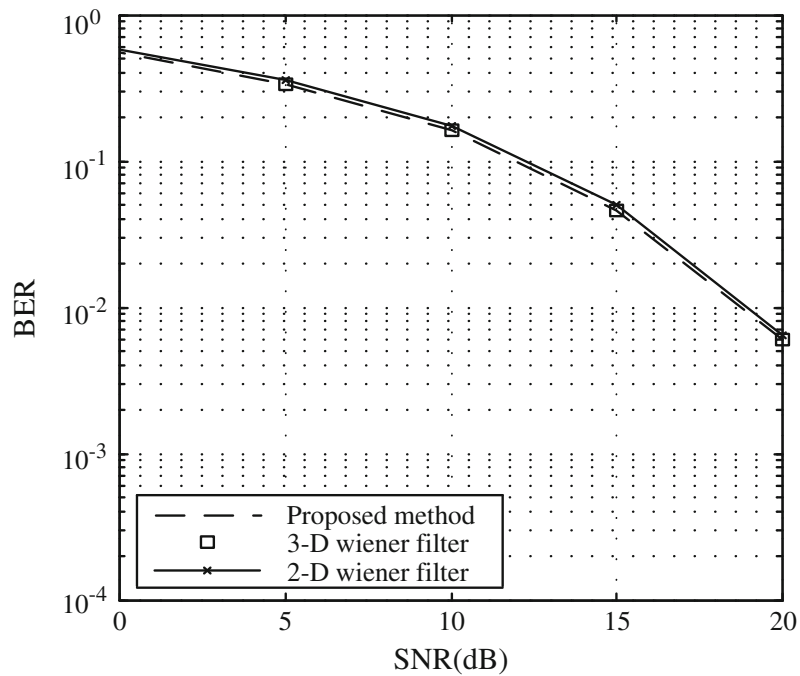

Fig. 10 Performance of BER: 64QAM, 120km/h

The wireless channel changes nonlinearly and drastically when the users move at $350 \mathrm{~km} / \mathrm{h}$. Figures 11, 12, 13 prove our established theories in Sect. 3.1 again and show that in this situation, the performance of Wiener filter with three orders is much better than that of Wiener filter with two orders since it can be observed that there are about 7.5 dB SNR gain at 16QAM modulation and about $5 \mathrm{~dB}$ SNR gain at 64QAM modulation. Figures 11, 12, 13 again prove that when the users move at high speeds, Wiener filter with order higher than two is needed to estimate the wireless channel in order to ensure the system performance. It is noted that even at $350 \mathrm{~km} / \mathrm{h}$, our proposed simplified Wiener filter technique is almost equal to the original Wiener filter without simplification. 


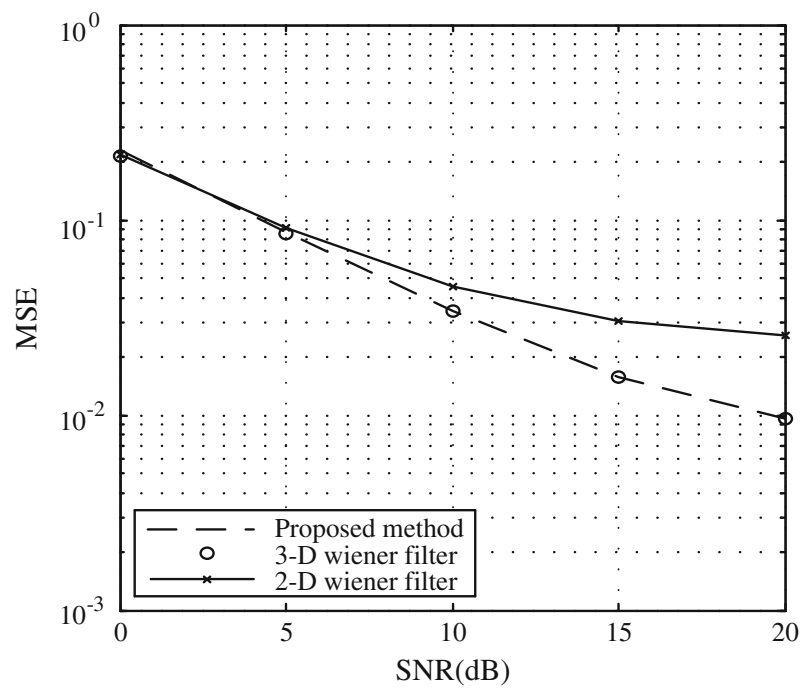

Fig. 11 Performance of MSE: 16QAM, $350 \mathrm{~km} / \mathrm{h}$

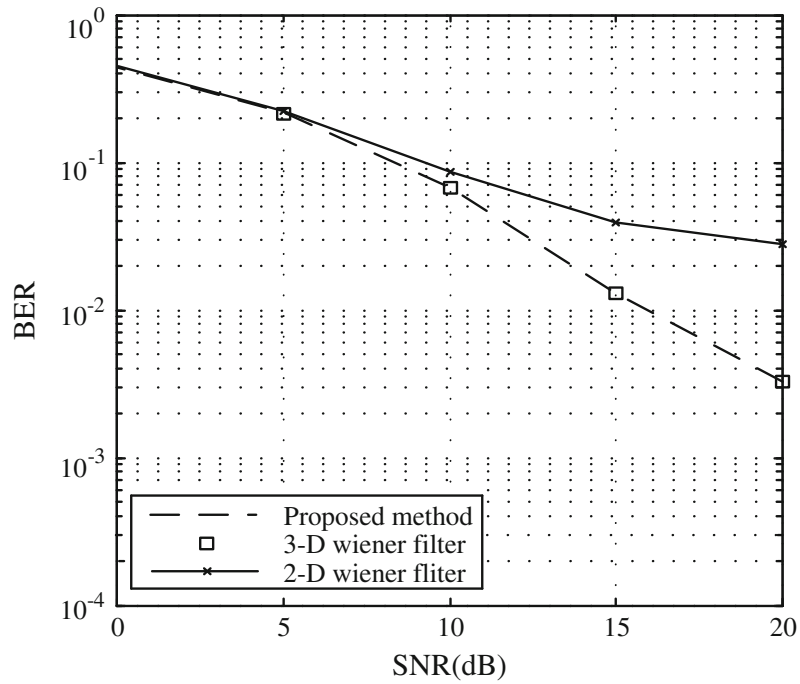

Fig. 12 Performance of BER: 16QAM, $350 \mathrm{~km} / \mathrm{h}$

Finally, the complete LTE uplink system with turbo coding is simulated in order to evaluate the performance of the proposed technique in practical systems which include coding. Figure 14 illustrates the performance of block error rate (BLER) with regard to SNR. Usually, for coded transmissions, BLER instead of BER is adopted to measure the performance. The details of the block considered in the LTE system are shown in Table 4 [17,18].

It is observed that Fig. 14 demonstrates the advantage of Wiener filter with high orders and the effectiveness of our proposed simplified technique once again. 


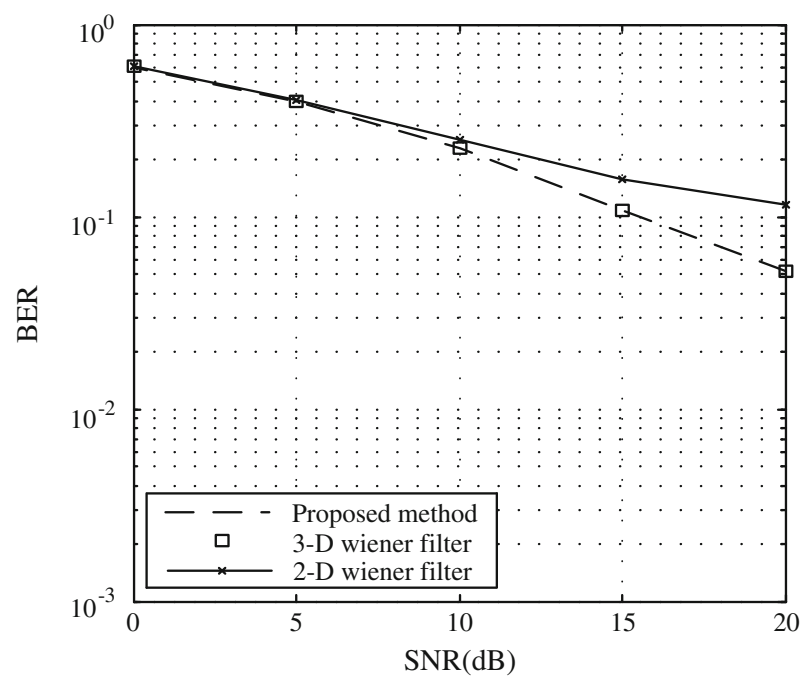

Fig. 13 Performance of BER: 64QAM, $350 \mathrm{~km} / \mathrm{h}$

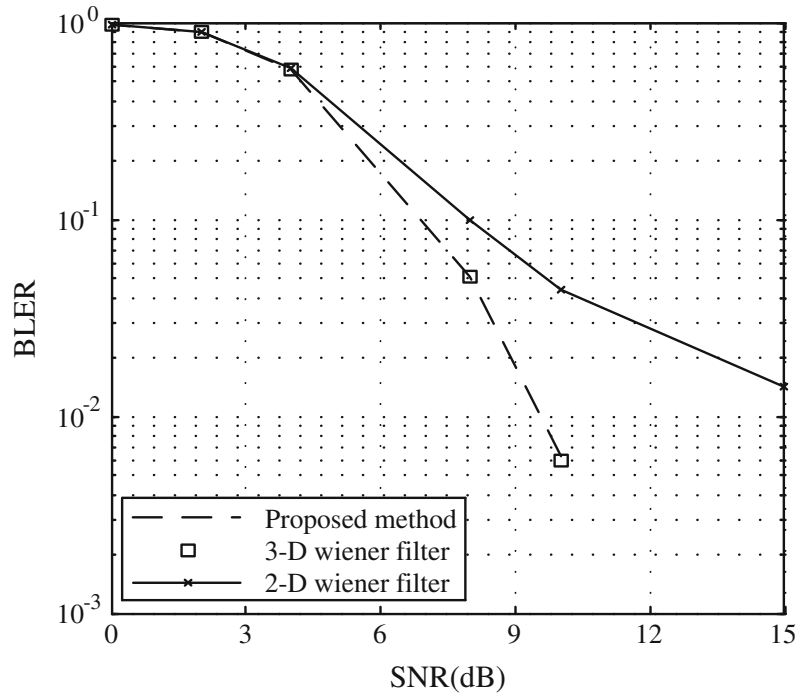

Fig. 14 Performance of BLER: 16QAM, $350 \mathrm{~km} / \mathrm{h}$

Table 4 The details of the block considered
Resource blocks (RB)

Modulation scheme 16QAM

Rate of turbo

$1 / 2$

Transmission block size (TBS) 


\section{Conclusion}

In this paper, we have mainly focused on the Wiener filter channel interpolation method that is implementable in practice. First, we analyzed the reason that Wiener filter with two orders lose its efficiency in high-speed environments. Then, the relationships among Wiener filter with different orders and their application scenarios were analyzed, based on which an extra pilot symbols selection criterion to complete Wiener filter higher than two orders in high-speed environments was investigated and a pilot symbol design criterion applicable to channel estimation in high-speed environments has been proposed. In the end, a simplified Wiener filter technique according to the signal correlation and the properties of Bessel function has been proposed. Extensive simulation results have demonstrated the effectiveness of our proposed theories and pilot design criterion. The performance difference between our proposed simplified Wiener filter technique and the original Wiener filter without simplification is trivial whereas the computational complexity is significantly reduced. Our proposed theories, criterion and technique have a potential value for practical implementation in the upcoming high-speed mobile wireless communication systems and can be readily applied to these systems.

Acknowledgments This work was supported in part by the National Science Foundation of China under Grant number 61032002, 60902026 and 60972029, the Chinese Important National Science \& Technology Specific Projects under Grant 2011ZX03001-007-01 and the Program for New Century Excellent Talents in University, NCET-11-0058.

Open Access This article is distributed under the terms of the Creative Commons Attribution License which permits any use, distribution, and reproduction in any medium, provided the original author(s) and the source are credited.

\section{Appendix A: The Derivation of MSE of the Simplified Wiener Filter Technique}

The MSE of channel estimation is given by

$$
\begin{aligned}
\varepsilon_{p} & =E\left\{\left|H_{p}-\hat{H}_{p}\right|^{2}\right\} \\
& =E\left\{H_{p} H_{p}^{*}\right\}-E\left\{H_{p} \hat{\mathbf{P}}^{H}\right\} \mathbf{C}_{p}^{H}-\mathbf{C}_{p} E\left\{\hat{\mathbf{P}} H_{p}^{*}\right\}+\mathbf{C}_{p} E\left\{\hat{\mathbf{P}} \hat{\mathbf{P}}^{H}\right\} \mathbf{C}_{p}^{H}
\end{aligned}
$$

Let $M=3$ in (2) and substituting the resulting $\hat{\mathbf{C}}_{p \text {, wiener }}$ into (17), then MMSE can be expressed as

$$
\varepsilon_{3 D, p}=1-\left(w_{3 D, 0} E\left\{\hat{H}_{p_{1}} H_{p}^{*}\right\}+w_{3 D, 1} E\left\{\hat{H}_{p_{2}} H_{p}^{*}\right\}+w_{3 D, 2} E\left\{\hat{H}_{p_{3}} H_{p}^{*}\right\}\right) .
$$

According to Table 1 and substituting the approximated Bessel function, which is expressed in (19), into (11)-(13) and continuing the derivation, it can be obtained that the estimation error for the coefficients of Wiener filter is also $\Delta$ order of magnitude and the result is given by (20), where $\Delta$ represents the approximation error in order of magnitude.

$$
\begin{gathered}
E\left\{H_{p_{i}} \hat{H}_{p_{j}}^{*}\right\}_{\text {proposed }}=E\left\{H_{p_{i}} \hat{H}_{p_{j}}^{*}\right\}_{\text {original }} \pm \Delta, \\
\left(w_{3 D, i}\right)_{\text {proposed }}=\left(w_{3 D, i}\right)_{\text {original }} \pm \Delta, \quad i=1,2,3 .
\end{gathered}
$$


Finally, substituting (20) into (18) and after some manipulations the MMSE of our proposed simplified Wiener filter technique can be expressed as

$$
\left(\varepsilon_{3 D, p}\right)_{\text {proposed }}=\left(\varepsilon_{3 D, p}\right)_{\text {original }} \mp \Delta\left(\sum_{i=0}^{2} w_{3 D, i}+\sum_{i=1}^{3} E\left\{\hat{H}_{p_{i}} H_{p}^{*}\right\} \pm 3 \times \Delta^{2}\right) .
$$

It is observed that if $\Delta>10^{-3}$, the accuracy of the approximation is not enough to guarantee the accuracy of channel estimation. If $\Delta<10^{-3}$, more $l$ in $F\left(q_{i-j}\right)$ must be selected to guarantee the accuracy of the approximation, in which case the computational complexity will be increased. Only when $\Delta=10^{-3}$, a compromise can be made between the accuracy of the approximation and the computational complexity, and at the same time guarantees the accuracy of channel estimation.

\section{Appendix B: Details of the Simplified Wiener Filter Technique in the Simulation}

In the considered LTE system, when velocity is $350 \mathrm{~km} / \mathrm{h}, f_{D}=745.3704 \mathrm{~Hz}$ when velocity is $120 \mathrm{~km} / \mathrm{h}, f_{D}=255.5556 \mathrm{~Hz}$. The symbol period $T=7.1354 \times 10^{-5} \mathrm{~s}$. Substituting the above $f_{D}, T$ and $(i-j)$ into $q_{i-j}$, we can obtain

$$
q_{i-j}=\left\{\begin{array}{ll}
-5.4719, & \text { when velocity is } 350 \mathrm{~km} / \mathrm{h} \\
-0.6404, & \text { when velocity is } 120 \mathrm{~km} / \mathrm{h}
\end{array} .\right.
$$

According to the proposed selection criterion in Table $1, l$ is thus obtained as

$$
l=\left\{\begin{array}{ll}
6, & \text { when velocity is } 350 \mathrm{~km} / \mathrm{h} \\
2, & \text { when velocity is } 120 \mathrm{~km} / \mathrm{h}
\end{array} .\right.
$$

Substituting (23) into (8) and then substituting the resulting (8) into (11)-(13), after a few tedious, but otherwise straightforward, algebraic manipulations, the simplified closed-form expression for Wiener filter can be obtained as follows

(a) When velocity is $350 \mathrm{~km} / \mathrm{h}$,

(a.1) The simplified expressions for the coefficients of symbols 1-3, 5-7 are obtained as follows,

- The corresponding determinant of Wiener filter with three orders is expressed as

$$
\begin{aligned}
|\mathbf{A}|= & \left(-372 q-25947 q^{2}-1.0518 e 6 q^{3}-2.6759 e 7 q^{4}-4.5887 e 8 q^{5}-5.6187 e 9 q^{6}\right. \\
& \left.-5.1370 e 10 q^{7}-3.6035 e 11 q^{8}-1.9371 e 12 q^{9}-7.7513 e 12 q^{10}\right)\left(\sigma^{2}\right) \\
& -9.4864 e 4 q^{3}-4.9013 e 6 q^{4}-1.1965 e 8 q^{5}-1.1840 q^{6}-1.9154 e 10 q^{7} \\
& -1.4876 e 11 q^{8}-8.4880 e 11 q^{9}
\end{aligned}
$$

- The simplified expressions for the coefficients of symbol $1 w 1_{3 D, j}, j=0,1,2$ are expressed as

$$
\begin{aligned}
w 1_{3 D, 0}= & \frac{1}{|\mathbf{A}|}\left[\left(-244 q-1.8488 e 4 q^{2}-7.4655 e 5 q^{3}-1.7981 e 7 q^{4}-2.8542 e 8 q^{5}\right.\right. \\
& \left.-3.2049 e 9 q^{6}-2.6765 e 10 q^{7}\right)\left(\sigma^{2}\right)-6.468 e 4 q^{3}-3.0939 e 6 q^{4}-6.9851 e 7 q^{5} \\
& \left.-9.7687 e 8 q^{6}-9.4905 e 9 q^{7}-6.7585 e 10 q^{8}\right]
\end{aligned}
$$




$$
\begin{aligned}
w 1_{3 D, 1}= & \frac{1}{|\mathbf{A}|}\left[\left(-148 q-8040 q^{2}-2.1371 e 5 q^{3}-3.3724 e 6 q^{4}-3.5066 e 7 q^{5}\right.\right. \\
& \left.-2.5795 e 8 q^{6}\right)\left(\sigma^{2}\right)-3.388 e 4 q^{3}-1.9368 e 6 q^{4}-5.2686 e 7 q^{5}-8.9397 e 8 q^{6} \\
& \left.-1.0626 e 10 q^{7}-9.3602 e 10 q^{8}-6.0826 e 11 q^{9}\right], \\
w 1_{3 D, 2}= & \frac{1}{|\mathbf{A}|}\left[\left(20 q+157 q^{2}-7.0278 e 3 q^{3}-1.2764 e 5 q^{4}-9.8546 e 5 q^{5}-4.5708 e 6 q^{6}\right.\right. \\
& \left.-7.3947 e 6 q^{7}\right)\left(\sigma^{2}\right)+3.696 e 3 q^{3}+1.2936 e 5 q^{4}+1.935 e 6 q^{5}+1.7256 e 7 q^{6} \\
& \left.+1.0597 e 8 q^{7}\right] .
\end{aligned}
$$

- The simplified expressions for the coefficients of symbol $2 w 2_{3 D, j}, j=0,1,2$ are expressed as

$$
\begin{aligned}
w 2_{3 D, 0}= & \frac{1}{|\mathbf{A}|}\left[\left(-214 q-1.5226 e 4 q^{2}-5.5935 e 5 q^{3}-1.2224 e 7 q^{4}-1.7598 e 8 q^{5}\right.\right. \\
& \left.-1.7922 e 9 q^{6}-1.3568 e 10 q^{7}\right) \sigma^{2}-3.8808 e 4 q^{3}-1.7334 e 6 q^{4}-3.6444 e 7 q^{5} \\
& \left.-4.7292 e 8 q^{6}-4.2477 e 9 q^{7}-2.7756 e 10 q^{8}\right], \\
w 2_{3 D, 1}= & \frac{1}{|\mathbf{A}|}\left[\left(-142 q-6.3335 e 3 q^{2}-1.4745 e 5 q^{3}-2.0582 e 6 q^{4}-1.8957 e 7 q^{5}\right.\right. \\
& \left.-1.2354 e 8 q^{6}\right) \sigma^{2}-6.0984 e 4 q^{3}-3.3338 e 6 q^{4}-8.6434 e 7 q^{5}-1.3929 e 9 q^{6} \\
& \left.-1.5660 e 10 q^{7}-1.2996 e 11 q^{8}-7.9491 e 11 q^{9}\right], \\
w 2_{3 D, 2}= & \frac{1}{|\mathbf{A}|}\left[\left(-16 q-1668 q^{2}-4.0679 e 4 q^{3}-4.5995 e 5 q^{4}-3.0827 e 6 q^{5}\right.\right. \\
& \left.-1.3898 e 7 q^{6}-3.2309 e 7 q^{7}\right) \sigma^{2}+4928 q^{3}+1.6591 e 5 q^{4}+2.3710 e 6 q^{5} \\
& \left.+2.0020 e 7 q^{6}+1.5818 e 8 q^{7}\right] .
\end{aligned}
$$

- The simplified expressions for the coefficients of symbol $3 w 3_{3 D, j}, j=0,1,2$ are expressed as

$$
\begin{aligned}
w 3_{3 D, 0}= & \frac{1}{|\mathbf{A}|}\left[\left(-184 q-12468 q^{2}-4.1479 e 5 q^{3}-8.1801 e 6 q^{4}-1.0626 e 8 q^{5}\right.\right. \\
& \left.-9.7652 e 8 q^{6}-6.6641 e 9 q^{7}\right) \sigma^{2}-1.7248 e 4 q^{3}-7.2729 e 5 q^{4}-1.4384 e 7 q^{5} \\
& \left.-1.75 e 8 q^{6}-1.4685 e 9 q^{7}-8.8663 e 9 q^{8}\right], \\
w 3_{3 D, 1}= & \frac{1}{|\mathbf{A}|}\left[\left(-136 q-4988 q^{2}-1.0018 e 5 q^{3}-1.2225 e 6 q^{4}-9.8842 e 6 q^{5}\right.\right. \\
& \left.-5.6604 e 7 q^{6}\right) \sigma^{2}-8.1312 e 4 q^{3}-4.2960 e 6 q^{4}-1.0736 e 8 q^{5}-1.6644 e 9 q^{6} \\
& \left.-1.7956 e 10 q^{7}-1.4250 e 11 q^{8}-8.3097 e 11 q^{9}\right], \\
w 3_{3 D, 2}= & \frac{1}{|\mathbf{A}|}\left[\left(-52 q-3371 q^{2}-7.3940 e 4 q^{3}-8.4680 e 5 q^{4}-6.1039 e 6 q^{5}\right.\right. \\
& \left.-3.0623 e 7 q^{6}-9.9105 e 7 q^{7}\right) \sigma^{2}+3.6960 e 3 q^{3}+1.2197 e 5 q^{4}+1.7095 e 6 q^{5} \\
& \left.+1.4105 e 7 q^{6}+7.8726 e 7 q^{7}\right] .
\end{aligned}
$$


- The simplified expressions for the coefficients of symbol $5 w 5_{3 D, j}, j=0,1,2$ are expressed as

$$
\begin{aligned}
w 5_{3 D, 0}= & \frac{1}{|\mathbf{A}|}\left[\left(-124 q-8108 q^{2}-2.2079 e 5 q^{3}-3.4883 e 6 q^{4}-3.6242 e 7 q^{5}\right.\right. \\
& \left.-2.6644 e 8 q^{6}-1.4432 e 9 q^{7}\right) \sigma^{2}+1.2936 e 4 q^{3}+5.0666 e 5 q^{4}+9.2734 e 6 q^{5} \\
& \left.+1.0459 e 8 q^{6}+8.1586 e 8 q^{7}+4.4937 e 9 q^{8}\right], \\
w 5_{3 D, 1}= & \frac{1}{|\mathbf{A}|}\left[\left(-124 q-3308 q^{2}-4.6697 e 4 q^{3}-4.0914 e 5 q^{4}-2.4238 e 6 q^{5}\right.\right. \\
& \left.-1.0295 e 7 q^{6}\right) \sigma^{2}-1.0164 e 5 q^{3}-5.2006 e 6 q^{4}-1.2588 e 8 q^{5}-1.9014 e 9 q^{6} \\
& \left.-2.0111 e 10 q^{7}-1.5725 e 11 q^{8}-9.09 e 11 q^{9}\right], \\
w 5_{3 D, 2}= & \frac{1}{|\mathbf{A}|}\left[\left(-124 q-6843 q^{2}-1.6135 e 5 q^{3}-2.2351 e 6 q^{4}-2.0487 e 7 q^{5}\right.\right. \\
& \left.-1.3320 e 8 q^{6}-6.3026 e 8 q^{7}\right) \sigma^{2}-6160 q^{3}-2.0739 e 5 q^{4}-3.0419 e 6 q^{5} \\
& \left.-2.6628 e 7 q^{6}-1.5877 e 8 q^{7}\right] .
\end{aligned}
$$

- The simplified expressions for the coefficients of symbol $6 w 6_{3 D, j}, j=0,1,2$ are expressed as

$$
\begin{aligned}
w 6_{3 D, 0}= & \frac{1}{|\mathbf{A}|}\left[\left(-94 q-6.3255 e 3 q^{2}-1.5790 e 5 q^{3}-2.2225 e 6 q^{4}-2.0458 e 7 q^{5}\right.\right. \\
& \left.-1.3315 e 8 q^{6}-6.3023 e 8 q^{7}\right) \sigma^{2}+2.1560 e 4 q^{3}+8.3365 e 5 q^{4}+1.5101 e 7 q^{5} \\
& \left.+1.6996 e 8 q^{6}+1.3362 e 9 q^{7}+7.4272 e 9 q^{8}\right], \\
w 6_{3 D, 1}= & \frac{1}{|\mathbf{A}|}\left[\left(-118 q-2.9375 e 3 q^{2}-3.5259 e 4 q^{3}-2.5604 e 5 q^{4}-1.2497 e 6 q^{5}\right.\right. \\
& \left.-4.3851 e 6 q^{6}\right) \sigma^{2}-1.0164 e 5 q^{3}-5.2175 e 6 q^{4}-1.2718 e 8 q^{5}-1.9505 e 9 q^{6} \\
& \left.-2.1144 e 10 q^{7}-1.7102 e 11 q^{8}-1.0343 e 12 q^{9}\right], \\
w 6_{3 D, 2}= & \frac{1}{|\mathbf{A}|}\left[\left(-160 q-8828 q^{2}-2.2648 e 5 q^{3}-3.5136 e 6 q^{4}-3.6316 e 7 q^{5}\right.\right. \\
& \left.-2.6659 e 8 q^{6}-1.4434 e 9 q^{7}\right) \sigma^{2}-1.4784 e 4 q^{3}-5.1744 e 5 q^{4}-8.5026 e 6 q^{5} \\
& \left.-7.5739 e 7 q^{6}-4.8948 e 8 q^{7}\right] .
\end{aligned}
$$

- The simplified expressions for the coefficients of symbol $7 w 7_{3 D, j}, j=0,1,2$ are expressed as

$$
\begin{aligned}
w 7_{3 D, 0}= & \frac{1}{|\mathbf{A}|}\left[\left(-64 q-4688 q^{2}-1.1013 e 5 q^{3}-1.3904 e 6 q^{4}-1.1301 e 7 q^{5}\right.\right. \\
& \left.-6.4668 e 7 q^{6}-2.6036 e 8 q^{7}\right) \sigma^{2}+2.5872 e 4 q^{3}+1.0047 e 6 q^{4}+1.8388 e 7 q^{5} \\
& \left.+2.1174 e 8 q^{6}+1.7279 e 9 q^{7}+1.0051 e 10 q^{8}\right]
\end{aligned}
$$




$$
\begin{aligned}
w 7_{3 D, 1}= & \frac{1}{|\mathbf{A}|}\left[\left(-112 q-2856 q^{2}-3.2203 e 4 q^{3}-2.1278 e 5 q^{4}-9.2568 e 5 q^{5}\right.\right. \\
& \left.-2.8496 e 6 q^{6}\right) \sigma^{2}-9.4864 e 4 q^{3}-4.9487 e 6 q^{4}-1.2329 e 8 q^{5}-1.9533 e 9 q^{6} \\
& \left.-2.2115 e 10 q^{7}-1.8881 e 11 q^{8}-1.2191 e 12 q^{9}\right], \\
w 7_{3 D, 2}= & \frac{1}{|\mathbf{A}|}\left[\left(-196 q-11123 q^{2}-3.1392 e 5 q^{3}-5.4383 e 6 q^{4}-6.2956 e 7 q^{5}\right.\right. \\
& \left.-5.1803 e 8 q^{6}-3.1624 e 9 q^{7}\right) \sigma^{2}-2.5872 e 4 q^{3}-9.5726 e 5 q^{4}-1.6076 e 7 q^{5} \\
& \left.-1.6507 e 8 q^{6}-1.1731 e 9 q^{7}\right] .
\end{aligned}
$$

(a.2) The simplified expressions for the coefficients of symbols 8-10, 12-13 are obtained as follows,

- The corresponding determinant of Wiener filter with three orders is expressed as

$$
\begin{aligned}
|\mathbf{A}|= & \left(-316 q-18723 q^{2}-6.2132 e 5 q^{3}-1.2854 e 7 q^{4}-1.7994 e 8 q^{5}-1.8071 e 9 q^{6}\right. \\
& \left.-1.3595 e 10 q^{7}-7.8627 e 10 q^{8}-3.4886 e 11 q^{9}\right)\left(\sigma^{2}\right)-4.41 e 4 q^{3}-1.9355 e 6 q^{4} \\
& -4.0137 e 7 q^{5}-5.1459 e 8 q^{6}-4.5729 e 9 q^{7}-2.9775 e 10 q^{8}-1.4176 e 11 q^{9}
\end{aligned}
$$

- The simplified expressions for the coefficients of symbol $8 w 8_{3 D, j}, j=0,1,2$ are expressed as

$$
\begin{aligned}
w 8_{3 D, 0}= & \frac{1}{|\mathbf{A}|}\left[\left(-162 q-7.3575 e 3 q^{2}-1.6092 e 5 q^{3}-2.1685 e 6 q^{4}-1.9669 e 7 q^{5}\right) \sigma^{2}\right. \\
& \left.-1.1340 e 4 q^{3}-3.4398 e 5 q^{4}-4.6690 e 6 q^{5}-3.8436 e 7 q^{6}-2.1792 e 8 q^{7}\right] \\
w 8_{3 D, 1}= & \frac{1}{|\mathbf{A}|}\left[\left(-92 q-2.076 e 3 q^{2}-2.1043 e 4 q^{3}-1.2826 e 5 q^{4}-5.2650 e 5 q^{5}\right) \sigma^{2}\right. \\
& -5.04 e 4 q^{3}-2.1952 e 6 q^{4}-4.5748 e 7 q^{5}-6.0778 e 8 q^{6}-5.7851 e 9 q^{7} \\
& \left.-4.1621 e 10 q^{8}-2.2691 e 11 q^{9}\right], \\
w 8_{3 D, 2}= & \frac{1}{|\mathbf{A}|}\left[\left(-62 q-3.6375 e 3 q^{2}-7.2105 e 4 q^{3}-7.8451 e 5 q^{4}-5.5385 e 6 q^{5}\right.\right. \\
& \left.-2.7596 e 7 q^{6}-9.8408 e 7 q^{7}\right) \sigma^{2}+1.764 e 4 q^{3}+6.0368 e 5 q^{4}+9.8391 e 6 q^{5} \\
& \left.+1.0149 e 8 q^{6}+7.4479 e 8 q^{7}+3.9855 e 9 q^{8}\right] .
\end{aligned}
$$

- The simplified expressions for the coefficients of symbol $9 w 9_{3 D, j}, j=0,1,2$ are expressed as

$$
\begin{aligned}
w 9_{3 D, 0}= & \frac{1}{|\mathbf{A}|}\left[\left(-128 q-5644 q^{2}-1.1134 e 5 q^{3}-1.3270 e 6 q^{4}-1.0601 e 7 q^{5}\right) \sigma^{2}\right. \\
& \left.-6.3 e 3 q^{3}-1.8025 e 5 q^{4}-2.2431 e 6 q^{5}-1.6668 e 7 q^{6}-8.4542 e 7 q^{7}\right],(47) \\
w 9_{3 D, 1}= & \frac{1}{|\mathbf{A}|}\left[\left(-100 q-2375 q^{2}-2.8728 e 4 q^{3}-2.1571 e 5 q^{4}-1.0939 e 6 q^{5}\right) \sigma^{2}\right. \\
& -5.25 e 4 q^{3}-2.2575 e 6 q^{4}-4.6127 e 7 q^{5}-5.9268 e 8 q^{6}-5.3795 e 9 q^{7} \\
& \left.-3.6436 e 10 q^{8}-1.8447 e 11 q^{9}\right],
\end{aligned}
$$




$$
\begin{aligned}
w 9_{3 D, 2}= & \frac{1}{|\mathbf{A}|}\left[\left(-88 q-4.904 e 3 q^{2}-1.0561 e 5 q^{3}-1.3015 e 6 q^{4}-1.0527 e 7 q^{5}\right.\right. \\
& \left.-6.0236 e 7 q^{6}-2.5266 e 8 q^{7}\right) \sigma^{2}+1.47 e 4 q^{3}+5.0225 e 5 q^{4}+8.1106 e 6 q^{5} \\
& \left.+8.1626 e 7 q^{6}+5.7478 e 8 q^{7}+2.9165 e 9 q^{8}\right] .
\end{aligned}
$$

- The simplified expressions for the coefficients of symbol $10 w 10_{3 D, j}, j=0,1,2$ are expressed as

$$
\begin{aligned}
w 10_{3 D, 0}= & \frac{1}{|\mathbf{A}|}\left[\left(-94 q-4.1655 e 3 q^{2}-7.5580 e 4 q^{3}-7.9714 e 5 q^{4}-5.5680 e 6 q^{5}\right) \sigma^{2}\right. \\
& \left.-2.52 e 3 q^{3}-6.944 e 4 q^{4}-8.0960 e 5 q^{5}-5.5376 e 6 q^{6}-2.5547 e 7 q^{7}\right],
\end{aligned}
$$

$$
\begin{aligned}
w 10_{3 D, 1}= & \frac{1}{|\mathbf{A}|}\left[\left(-108 q-2.916 e 3 q^{2}-4.3083 e 4 q^{3}-3.9133 e 5 q^{4}-2.3686 e 6 q^{5}\right) \sigma^{2}\right. \\
& -5.04 e 4 q^{3}-2.1728 e 6 q^{4}-4.4320 e 7 q^{5}-5.6239 e 8 q^{6}-4.9812 e 9 q^{7} \\
& \left.-3.2540 e 10 q^{8}-1.5670 e 11 q^{9}\right], \\
w 10_{3 D, 2}= & \frac{1}{|\mathbf{A}|}\left[\left(-114 q-6.3255 e 3 q^{2}-1.5128 e 5 q^{3}-2.1159 e 6 q^{4}-1.9480 e 7 q^{5}\right.\right. \\
& \left.-1.2689 e 8 q^{6}-6.1136 e 8 q^{7}\right) \sigma^{2}+8.82 e 3 q^{3}+3.0674 e 5 q^{4}+5.0221 e 6 q^{5} \\
& \left.+5.0717 e 7 q^{6}+3.5411 e 8 q^{7}+1.7712 e 9 q^{8}\right] .
\end{aligned}
$$

- The simplified expressions for the coefficients of symbol $12 w 12_{3 D, j}, j=0,1,2$ are expressed as

$$
\begin{aligned}
w 12_{3 D, 0}= & \frac{1}{|\mathbf{A}|}\left[\left(-26 q-1.5055 e 3 q^{2}-2.7497 e 4 q^{3}-2.5007 e 5 q^{4}-1.3858 e 6 q^{5}\right) \sigma^{2}\right. \\
& \left.+1.26 e 3 q^{3}+3.4580 e 4 q^{4}+3.8945 e 5 q^{5}+2.5248 e 6 q^{6}+1.0917 e 7 q^{7}\right]
\end{aligned}
$$

$$
\begin{aligned}
w 12_{3 D, 1}= & \frac{1}{|\mathbf{A}|}\left[\left(-124 q-4.82 e 3 q^{2}-9.9281 e 4 q^{3}-1.2199 e 6 q^{4}-9.8795 e 6 q^{5}\right) \sigma^{2}\right. \\
& -3.36 e 4 q^{3}-1.5232 e 6 q^{4}-3.2662 e 7 q^{5}-4.3234 e 8 q^{6}-3.9608 e 9 q^{7} \\
& \left.-2.6571 e 10 q^{8}-1.3018 e 11 q^{9}\right]
\end{aligned}
$$

$$
\begin{aligned}
w 12_{3 D, 2}= & \frac{1}{|\mathbf{A}|}\left[\left(-166 q-9.9455 e 3 q^{2}-2.975 e 5 q^{3}-5.2684 e 6 q^{4}-6.1442 e 7 q^{5}\right.\right. \\
& \left.-5.0683 e 8 q^{6}-3.1075 e 9 q^{7}\right) \sigma^{2}-1.176 e 4 q^{3}-4.4688 e 5 q^{4}-8.0016 e 6 q^{5} \\
& \left.-8.8051 e 7 q^{6}-6.6717 e 8 q^{7}-3.6602 e 9 q^{8}\right] .
\end{aligned}
$$

- The simplified expressions for the coefficients of symbol $13 w 13_{3 D, j}, j=0,1,2$ are expressed as

$$
\begin{aligned}
w 13_{3 D, 0}= & \frac{1}{|\mathbf{A}|}\left[\left(8 q-120 q^{2}-6.6451 e 3 q^{3}-7.8118 e 4 q^{4}-4.6460 e 5 q^{5}\right) \sigma^{2}\right. \\
& \left.+1.26 e 3 q^{3}+3.577 e 4 q^{4}+4.1772 e 5 q^{5}+2.8229 e 6 q^{6}+1.2824 e 7 q^{7}\right],
\end{aligned}
$$




$$
\begin{aligned}
w 13_{3 D, 1}= & \frac{1}{|\mathbf{A}|}\left[\left(-132 q-6.231 e 3 q^{2}-1.4706 e 5 q^{3}-2.0574 e 6 q^{4}-1.8956 e 7 q^{5}\right) \sigma^{2}\right. \\
& -1.89 e 4 q^{3}-8.967 e 5 q^{4}-2.0174 e 7 q^{5}-2.8068 e 8 q^{6}-2.7090 e 9 q^{7} \\
& \left.-1.9198 e 10 q^{8}-9.9403 e 10 q^{9}\right], \\
w 13_{3 D, 2}= & \frac{1}{|\mathbf{A}|}\left[\left(-192 q-1.23 e 4 q^{2}-4.092 e 5 q^{3}-8.0754 e 6 q^{4}-1.0494 e 8 q^{5}\right.\right. \\
& \left.-9.646 e 8 q^{6}-6.594 e 9 q^{7}\right) \sigma^{2}-2.646 e 4 q^{3}-1.0745 e 6 q^{4}-2.0602 e 7 q^{5} \\
& \left.-2.4345 e 8 q^{6}-1.9874 e 9 q^{7}-1.1836 e 10 q^{8}\right] .
\end{aligned}
$$

(b) When velocity is $120 \mathrm{~km} / \mathrm{h}$,

(b.1) The simplified expressions for the coefficients of symbols 1-3, 5-7 are obtained as follows,

- The corresponding determinant of Wiener filter with three orders is expressed as

$$
|\mathbf{A}|=3 \sigma^{4}+\left(-372 q-25947 q^{2}-1.0518 e 6 q^{3}\right)\left(\sigma^{2}\right)-9.4864 e 4 q^{3}-4.9013 e 6 q^{4} .(59)
$$

- The simplified expressions for the coefficients of symbol $1 w 1_{3 D, j}, j=0,1,2$ are expressed as

$$
\begin{aligned}
w 1_{3 D, 0}= & \frac{1}{|\mathbf{A}|}\left[\left(1+q+0.25 q^{2}\right)\left(\sigma^{4}\right)+\left(-244 q-1.8488 e 4 q^{2}-7.4655 e 5 q^{3}\right)\left(\sigma^{2}\right)\right. \\
& \left.-6.468 e 4 q^{3}-3.0939 e 6 q^{4}\right] \\
w 1_{3 D, 1}= & \frac{1}{|\mathbf{A}|}\left[\left(1+9 q+20.25 q^{2}\right)\left(\sigma^{4}\right)+\left(-148 q-8040 q^{2}-2.1371 e 5 q^{3}\right)\left(\sigma^{2}\right)\right. \\
& \left.-3.388 e 4 q^{3}-1.9368 e 6 q^{4}\right] \\
w 1_{3 D, 2}= & \frac{1}{|\mathbf{A}|}\left[\left(1+100 q+2500 q^{2}\right)\left(\sigma^{4}\right)+\left(20 q+157 q^{2}-7.0278 e 3 q^{3}\right)\left(\sigma^{2}\right)\right. \\
& \left.+3.696 e 3 q^{3}+1.2936 e 5 q^{4}\right] .
\end{aligned}
$$

- The simplified expressions for the coefficients of symbol $2 w 2_{3 D, j}, j=0,1,2$ are expressed as

$$
\begin{aligned}
w 2_{3 D, 0}= & \frac{1}{|\mathbf{A}|}\left[\left(1+4 q+4 q^{2}\right)\left(\sigma^{4}\right)+\left(-214 q-1.5226 e 4 q^{2}-5.5935 e 5 q^{3}\right) \sigma^{2}\right. \\
& \left.-3.8808 e 4 q^{3}-1.7334 e 6 q^{4}\right] \\
w 2_{3 D, 1}= & \frac{1}{|\mathbf{A}|}\left[\left(1+4 q+4 q^{2}\right)\left(\sigma^{4}\right)+\left(-142 q-6.3335 e 3 q^{2}-1.4745 e 5 q^{3}\right) \sigma^{2}\right. \\
& \left.-6.0984 e 4 q^{3}-3.3338 e 6 q^{4}\right], \\
w 2_{3 D, 2}= & \frac{1}{|\mathbf{A}|}\left[\left(1+81 q+1.6403 e 3 q^{2}\right)\left(\sigma^{4}\right)+\left(-16 q-1668 q^{2}-4.0679 e 4 q^{3}\right) \sigma^{2}\right. \\
& \left.+4928 q^{3}+1.6591 e 5 q^{4}\right] .
\end{aligned}
$$


- The simplified expressions for the coefficients of symbol $3 w 3_{3 D, j}, j=0,1,2$ are expressed as

$$
\begin{aligned}
w 3_{3 D, 0}= & \frac{1}{|\mathbf{A}|}\left[\left(1+9 q+20.25 q^{2}\right)\left(\sigma^{4}\right)+\left(-184 q-12468 q^{2}-4.1479 e 5 q^{3}\right) \sigma^{2}\right. \\
& \left.-1_{1} 7248 e 4 q^{3}-7.2729 e 5 q^{4}\right] \\
w 3_{3 D, 1}= & \frac{1}{|\mathbf{A}|}\left[\left(1+q+0.25 q^{2}\right)\left(\sigma^{4}\right)+\left(-136 q-4988 q^{2}-1.0018 e 5 q^{3}\right) \sigma^{2}\right. \\
w 3_{3 D, 2}= & \frac{1}{|\mathbf{A}|}\left[\left(1+64 q+1.024 e 3 q^{2}\right)\left(\sigma^{4}\right)+\left(-52 q-3371 q^{2}-7.3940 e 4 q^{3}\right) \sigma^{2}\right. \\
& \left.+3.6960 e 3 q^{3}+1.2197 e 5 q^{4}\right] .
\end{aligned}
$$

- The simplified expressions for the coefficients of symbol $5 w 5_{3 D, j}, j=0,1,2$ are expressed as

$$
\begin{aligned}
w 5_{3 D, 0}= & \frac{1}{|\mathbf{A}|}\left[\left(1+25 q+156.25 q^{2}\right)\left(\sigma^{4}\right)+\left(-124 q-8108 q^{2}-2.2079 e 5 q^{3}\right) \sigma^{2}\right. \\
+ & \left.1.2936 e 4 q^{3}+5.0666 e 5 q^{4}\right], \\
w 5_{3 D, 1}= & \frac{1}{|\mathbf{A}|}\left[\left(1+q+0.25 q^{2}\right)\left(\sigma^{4}\right)+\left(-124 q-3308 q^{2}-4.6697 e 4 q^{3}\right) \sigma^{2}\right. \\
& \left.-1.0164 e 5 q^{3}-5.2006 e 6 q^{4}\right], \\
w 5_{3 D, 2}= & \frac{1}{|\mathbf{A}|}\left[\left(1+36 q+324 q^{2}\right)\left(\sigma^{4}\right)+\left(-124 q-6843 q^{2}-1.6135 e 5 q^{3}\right) \sigma^{2}\right. \\
& \left.-6160 q^{3}-2.0739 e 5 q^{4}\right] .
\end{aligned}
$$

- The simplified expressions for the coefficients of symbol $6 w 6_{3 D, j}, j=0,1,2$ are expressed as

$$
\begin{aligned}
w 6_{3 D, 0}= & \frac{1}{|\mathbf{A}|}\left[\left(1+36 q+324 q^{2}\right)\left(\sigma^{4}\right)+\left(-94 q-6.3255 e 3 q^{2}-1.5790 e 5 q^{3}\right) \sigma^{2}\right. \\
& \left.+2.1560 e 4 q^{3}+8.3365 e 5 q^{4}\right] \\
w 6_{3 D, 1}= & \frac{1}{|\mathbf{A}|}\left[\left(1+4 q+4 q^{2}\right)\left(\sigma^{4}\right)+\left(-118 q-2.9375 e 3 q^{2}-3.5259 e 4 q^{3}\right) \sigma^{2}\right. \\
& \left.-1.0164 e 5 q^{3}-5.2175 e 6 q^{4}\right] \\
w 6_{3 D, 2}= & \frac{1}{|\mathbf{A}|}\left[\left(1+25 q+156.25 q^{2}\right)\left(\sigma^{4}\right)+\left(-160 q-8828 q^{2}-2.2648 e 5 q^{3}\right) \sigma^{2}\right. \\
& \left.-1.4784 e 4 q^{3}-5.1744 e 5 q^{4}\right] .
\end{aligned}
$$

- The simplified expressions for the coefficients of symbol $7 w 7_{3 D, j}, j=0,1,2$ are expressed as

$$
\begin{aligned}
w 7_{3 D, 0}= & \frac{1}{|\mathbf{A}|}\left[\left(1+49 q+600.25 q^{2}\right)\left(\sigma^{4}\right)+\left(-64 q-4688 q^{2}-1.1013 e 5 q^{3}\right) \sigma^{2}\right. \\
& \left.+2.5872 e 4 q^{3}+1.0047 e 6 q^{4}\right]
\end{aligned}
$$




$$
\begin{aligned}
w 7_{3 D, 1}= & \frac{1}{|\mathbf{A}|}\left[\left(1+9 q+20.25 q^{2}\right)\left(\sigma^{4}\right)+\left(-112 q-2856 q^{2}-3.2203 e 4 q^{3}\right) \sigma^{2}\right. \\
& \left.-9.4864 e 4 q^{3}-4.9487 e 6 q^{4}\right], \\
w 7_{3 D, 2}= & \frac{1}{|\mathbf{A}|}\left[\left(1+16 q+64 q^{2}\right)\left(\sigma^{4}\right)+\left(-196 q-11123 q^{2}-3.1392 e 5 q^{3}\right) \sigma^{2}\right. \\
& \left.-2.5872 e 4 q^{3}-9.5726 e 5 q^{4}\right] .
\end{aligned}
$$

(b.2) The simplified expressions for the coefficients of symbols 8--10, 12--13 are obtained as follows,

- The corresponding determinant of Wiener filter with three orders is expressed as

$$
|\mathbf{A}|=3 \sigma^{4}+\left(-316 q-18723 q^{2}-6.2132 e 5 q^{3}\right)\left(\sigma^{2}\right)-4.41 e 4 q^{3}-1.9355 e 6 q^{4} .
$$

- The simplified expressions for the coefficients of symbol $8 w 8_{3 D, j}, j=0,1,2$ are expressed as

$$
\begin{aligned}
w 8_{3 D, 0}= & \frac{1}{|\mathbf{A}|}\left[\left(1+16 q+64 q^{2}\right)\left(\sigma^{4}\right)+\left(-162 q-7.3575 e 3 q^{2}-1.6092 e 5 q^{3}\right) \sigma^{2}\right. \\
& \left.-1.1340 e 4 q^{3}-3.4398 e 5 q^{4}\right] \\
w 8_{3 D, 1}= & \frac{1}{|\mathbf{A}|}\left[\left(1+9 q+20.25 q^{2}\right)\left(\sigma^{4}\right)+\left(-92 q-2.076 e 3 q^{2}-2.1043 e 4 q^{3}\right) \sigma^{2}\right. \\
& \left.-5.04 e 4 q^{3}-2.1952 e 6 q^{4}\right] \\
w 8_{3 D, 2}= & \frac{1}{|\mathbf{A}|}\left[\left(1+36 q+324 q^{2}\right)\left(\sigma^{4}\right)+\left(-62 q-3.6375 e 3 q^{2}-7.2105 e 4 q^{3}\right) \sigma^{2}\right. \\
& \left.+1.764 e 4 q^{3}+6.0368 e 5 q^{4}\right] .
\end{aligned}
$$

- The simplified expressions for the coefficients of symbol $9 w 9_{3 D, j}, j=0,1,2$ are expressed as

$$
\begin{aligned}
& w 9_{3 D, 0}=\frac{1}{|\mathbf{A}|}\left[\left(1+25 q+156.25 q^{2}\right)\left(\sigma^{4}\right)+\left(-128 q-5644 q^{2}-1.1134 e 5 q^{3}\right) \sigma^{2}\right. \\
& \left.-6.3 e 3 q^{3}-1.8025 e 5 q^{4}\right] \\
& w 9_{3 D, 1}=\frac{1}{|\mathbf{A}|}\left[\left(1+4 q+4 q^{2}\right)\left(\sigma^{4}\right)+\left(-100 q-2375 q^{2}-2.8728 e 4 q^{3}\right) \sigma^{2}\right. \\
& \left.-5.25 e 4 q^{3}-2.2575 e 6 q^{4}\right] \text {, } \\
& w 9_{3 D, 2}=\frac{1}{|\mathbf{A}|}\left[\left(1+25 q+156.25 q^{2}\right)\left(\sigma^{4}\right)+\left(-88 q-4.904 e 3 q^{2}-1.0561 e 5 q^{3}\right) \sigma^{2}\right. \\
& \left.+1.47 e 4 q^{3}+5.0225 e 5 q^{4}\right] \text {. }
\end{aligned}
$$

- The simplified expressions for the coefficients of symbol $10 w 10_{3 D, j}, j=0,1,2$ are expressed as

$$
\begin{aligned}
w 10_{3 D, 0}= & \frac{1}{|\mathbf{A}|}\left[\left(1+36 q+324 q^{2}\right)\left(\sigma^{4}\right)+\left(-94 q-41655 e 3 q^{2}-7.5580 e 4 q^{3}\right) \sigma^{2}\right. \\
& \left.-2.52 e 3 q^{3}-6.944 e 4 q^{4}\right],
\end{aligned}
$$




$$
\begin{aligned}
w 10_{3 D, 1}= & \frac{1}{|\mathbf{A}|}\left[\left(1+q+0.25 q^{2}\right)\left(\sigma^{4}\right)+\left(-108 q-2.916 e 3 q^{2}-4.3083 e 4 q^{3}\right) \sigma^{2}\right. \\
& \left.-5.04 e 4 q^{3}-2.1728 e 6 q^{4}\right], \\
w 10_{3 D, 2}= & \frac{1}{|\mathbf{A}|}\left[\left(1+16 q+64 q^{2}\right)\left(\sigma^{4}\right)+\left(-114 q-6.3255 e 3 q^{2}-1.5128 e 5 q^{3}\right) \sigma^{2}\right. \\
& \left.+8.82 e 3 q^{3}+3.0674 e 5 q^{4}\right] .
\end{aligned}
$$

- The simplified expressions for the coefficients of symbol $12 w 12_{3 D, j}, j=0,1,2$ are expressed as

$$
\begin{aligned}
& w 12_{3 D, 0}=\frac{1}{|\mathbf{A}|}\left[\left(1+64 q+1.024 e 3 q^{2}\right)\left(\sigma^{4}\right)+\left(-26 q-1.5055 e 3 q^{2}-2.7497 e 4 q^{3}\right) \sigma^{2}\right. \\
&+\left.1.26 e 3 q^{3}+3.458 e 4 q^{4}\right] \\
& w 12_{3 D, 1}= \frac{1}{|\mathbf{A}|}\left[\left(1+q+0.25 q^{2}\right)\left(\sigma^{4}\right)+\left(-124 q-4.82 e 3 q^{2}-9.9281 e 4 q^{3}\right) \sigma^{2}\right. \\
&\left.-3.36 e 4 q^{3}-1.5232 e 6 q^{4}\right] \\
& w 12_{3 D, 2}= \frac{1}{|\mathbf{A}|}\left[\left(1+4 q+4 q^{2}\right)\left(\sigma^{4}\right)+\left(-166 q-9.9455 e 3 q^{2}-2.975 e 5 q^{3}\right) \sigma^{2}\right. \\
&\left.-1.176 e 4 q^{3}-4.4688 e 5 q^{4}\right] .
\end{aligned}
$$

- The simplified expressions for the coefficients of symbol $13 w 13_{3 D, j}, j=0,1,2$ are expressed as

$$
\begin{aligned}
w 13_{3 D, 0}= & \frac{1}{|\mathbf{A}|}\left[\left(1+81 q+1.6403 e 3 q^{2}\right)\left(\sigma^{4}\right)+\left(8 q-120 q^{2}-6.6451 e 3 q^{3}\right) \sigma^{2}\right. \\
& \left.+1.26 e 3 q^{3}+3.577 e 4 q^{4}\right], \\
w 13_{3 D, 1}= & \frac{1}{|\mathbf{A}|}\left[\left(1+4 q+4 q^{2}\right)\left(\sigma^{4}\right)+\left(-132 q-6.231 e 3 q^{2}-1.4706 e 5 q^{3}\right) \sigma^{2}\right. \\
& \left.-1.89 e 4 q^{3}-8.967 e 5 q^{4}\right], \\
w 13_{3 D, 2}= & \frac{1}{|\mathbf{A}|}\left[\left(1+q+0.25 q^{2}\right)\left(\sigma^{4}\right)+\left(-192 q-1.23 e 4 q^{2}-4.092 e 5 q^{3}\right) \sigma^{2}\right. \\
& \left.-2.646 e 4 q^{3}-1.0745 e 6 q^{4}\right] .
\end{aligned}
$$

where $w i_{M D, m}, m=0,1, \ldots, M-1$ represent the coefficients of Wiener filter for the $i$ th symbol, $M D$ represents $M$ pilot symbols are adopted to complete interpolation at the time dimension and $q \triangleq-\pi^{2} f_{D}^{2} T^{2}$.

\section{References}

1. Sesia, S., Toufik, I., \& Baker, M. (2009). LTE-the UMTS long term evolution: From theory to practice. UK: Wiley.

2. Colieri, S., Ergen, M., \& Puri, A. (2002). A study of channel estimation in OFDM System. In Proceedings of the 56th vehicular technology conference (Vol. 2, pp. 894-898). 
3. He, C. L., \& Hao, L. (2009). Pilot-aided channel estimation techniques in OFDM system. In Proceedings of international conference on communication software and networks (pp. 143-146).

4. Khanshan, A. H., \& Amindavar, H. (2007). Performance evaluation of two-dimensional interpolations on OFDM channel estimation. In Proceedings of IEEE international conference in central Asia on internet (pp. 1-5).

5. Haykin, S. (2003). Adaptive filter theory. US: Pearson Education.

6. Dong, X. D., Lu, W. S., \& Soong, A. C. K. (2007). Linear interpolation in pilot symbol assisted channel estimation for OFDM. IEEE Transactions on Wireless Communications, 6(5), 1910-1920.

7. Li, Y. (2000). Pilot-symbol-aided channel estimation for OFDM in wireless systems. IEEE Transactions on Vehicular Technology, 49(4), 1207-1215.

8. Bader, F., \& Gonzalez, R. (2005). Analysis of the design of the optimum pilot pattern using different bi-dimensional interpolation filters. In Proceedings of the IEEE 16th international symposium on personal, indoor and mobile radio communications (Vol. 4, pp. 2484-2488).

9. Schafhuber, D., Matz, G., \& Hlawatsch, F. (2003). Adaptive wiener filters for time-varying channel estimation in wireless OFDM systems. In Proceedings of IEEE international conference on acoustics, speech, and signal processing (Vol. 4, pp. 688-691).

10. Zhang, J. H., \& Zhang, P. (2003). An improved 2-dimensional pilot-symbols assisted channel estimation in OFDM systems. In Proceedings of the 57th IEEE semiannual vehicular technology conference (Vol. 3, pp. 1595-1599).

11. Park, H. M., \& Lee, J. H. (2006). Estimation of time-variant channels for ofdm systems using Kalman and Wiener filters. In Proceedings of the IEEE 64th vehicular technology conference (pp. 1-5).

12. Hoeher, P., Kaiser, S., \& Robertson, P. (1997). Two-dimensional pilot-symbol-aided channel estimation by Wiener filtering. In Proceedings of IEEE international conference on acoustics, speech, and signal processing (Vol. 3, pp. 1845-1848).

13. Chen, W. W., \& Zhu, Q. (2007). Research on interpolation methods for channel estimation in the MIMO-OFDM systems. In Proceedings of international conference on wireless communications, networking and mobile computing (pp. 306-309).

14. Ma, Y. T., Liu. K. H., \& Duan, J. F. (2008). Research and implementation of channel estimation of digital radio receiver based on OFDM. In Proceedings of international conference on neural networks and signal processing, (pp. 104-108).

15. Zheng Y. R., \& Xiao C. S. (2009). Channel estimation for frequency-domain equalization of single-carrier broadband wireless communications. IEEE Transactions on Vehicular Technology, 58(2), $815-823$.

16. Van de Beek, J.-J., Edfors, O., \& Sandell, M. (1997) On channel estimation in OFDM systems. In Proceedings of the IEEE 45th vehicular technology conference, (Vol. 2, pp. 815-819).

17. 3GPP TS36.212, v8.7.0. (2009). Evolved universal terrestrial radio access (E-UTRA) multiplexing and channel coding.

18. 3GPP TS36.211, v8.7.0. (2009). Evolved universal terrestrial radio access (E-UTRA) physical channels and modulation.

\section{Author Biographies}

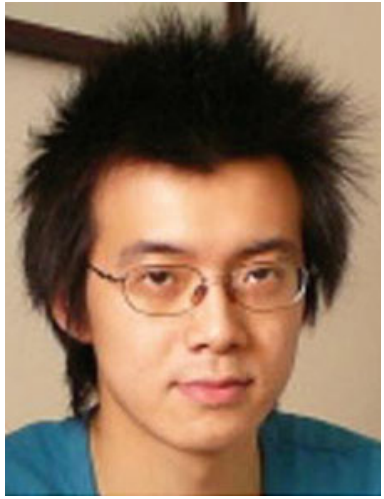

Ke Zhong was born in Chengdu, Sichuan, People's Republic of China in 1985. He receive his B.Eng. in College of Information Engineering from Chengdu University of Technology, Chengdu, China, in 2008, and now in successive postgraduate and doctoral programs of study in National Key Laboratory of Science and Technology on Communications, University of Electronic Science and Technology of China (UESTC), Chengdu, China, from 2008. His research interests include channel estimation in doubly-selective fading channels and MIMOOFDM systems. 

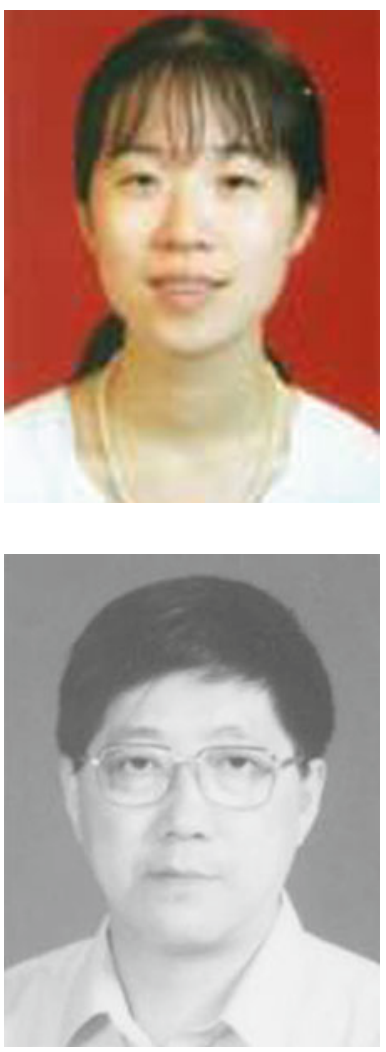

Xia Lei was born in MianYang, Sichuan, china, in 1975. In 2005, she received the Ph.D. degree from School of Communications and Information Engineering, University of Electronic Science and Technology of China (UESTC), Chengdu, China. Her research interests are in wireless communications, including new generation mobile communication, broadband wireless access and shortwave communication.

Shaoqian Li received the B.Eng. degree from University of Electronic Science and Technology of Xi' an, China, in 1982 and the M.Eng. degree from University of Electronic Science and Technology of China (UESTC), Sichuan, in 1984. Since July 1984, he has been a Faculty Member with the Institute of Communication and Information Engineering, UESTC, China, where he is a Professor, since 1997, and Ph.D. supervisor, since 2000. He is also the director of National Key Laboratory of Science and Technology on Communications in UESTC and is a Member of Telecommunication Subject with National High Technology R\&D Program of China (863 Program). His main fields of research interest include mobile communications systems $(3 \mathrm{G}$, Beyond $3 \mathrm{G}$ ), wireless communication (CDMA, OFDM, MIMO and etc.), integrate circuit in communication systems and radio/spatial resource management. 